Pacific

Journal of

Mathematics

\title{
DISCS AND THE MORERA PROPERTY
}

Josip GLobevnik and Edgar Lee Stout 


\title{
DISCS AND THE MORERA PROPERTY
}

\author{
Josip Globevnik And Edgar Lee Stout
}

In previous work, we have considered the problem of showing that a continuous function on a real hypersurface $\Gamma$ in $\mathbb{C}^{N}$ satisfies the tangential Cauchy-Riemann equations provided that its slices satisfy conditions of Morera type. For instance, these results imply that if $\Omega \subset \mathbb{C}^{N}$ is a bounded convex domain with smooth boundary, strictly convex at $z_{0} \in b D$, if $L_{0}$ is a complex line tangent to $b \Omega$ at $z_{0}$ and if $f$ is a continuous function on $b \Omega$ such that $\int_{L \cap b \Omega} f \omega=0$ for all complex lines $L$ close to $L_{0}$ which meet $\Omega$ and for all $(1,0)$ forms with constant coefficients, then $f$ is a CR function in a neighbourhood of $z_{0}$. This fails to hold if $L_{0}$ is a complex line that meets $\Omega$ even under much stronger assumption of holomorphic extendibility along complex lines. Indeed, let $\mathbb{B}$ be the open unit ball in $\mathbb{C}^{2}$, and define a function $f$ on $b \mathbb{B} \backslash\{z=0\}$ by $f(z, w)=1 / \bar{z}$. It is easy to verify that for each complex line $L$ close to the $z$-axis, $f \mid L \cap b \mathbb{B}$ has a continuous extension to $L \cap \overline{\mathbb{B}}$ which is holomorphic on $L \cap \mathbb{B}$, yet there is no open set in $b \mathbb{B}$ on which $f$ is a CR function. So to conclude that $f$ is a CR function one has to assume the holomorphic extension property for a larger family of analytic discs.

\section{Introduction and the main result.}

Let $\Delta$ be the open unit disc in $\mathbb{C}$. An analytic disc attached to a manifold $M \subset \mathbb{C}^{N}$ is a continuous map $\varphi: \bar{\Delta} \rightarrow \mathbb{C}^{N}$, holomorphic on $\Delta$ and such that $\varphi(b \Delta) \subset M$. A.E. Tumanov proved that if $f$ is a function of class $\mathcal{C}^{1}$ on a generic submanifold $M$ of $\mathbb{C}^{N}$, and $\varphi_{0}$ is an analytic disc attached to $M$ such that for all analytic discs $\varphi$, attached to $M$ and close to $\varphi_{0}$, the function $\zeta \rightarrow f(\varphi(\zeta))$ has a continuous extension from $b \Delta$ to $\bar{\Delta}$ which is holomorphic on $\Delta$, then $f$ is a CR function in a neighbourhood of $\varphi_{0}(b \Delta)$ [Tu1].

In the present paper we consider a similar problem with conditions of Morera type for continuous functions on real hypersurfaces. Let $\Omega \subset \mathbb{C}^{N}$ be a bounded domain with $\mathcal{C}^{2}$ boundary. A subset $D \subset \Omega$ is called a transversely embedded analytic disc if $D=V \cap \Omega$ where $V$ is a one dimensional complex submanifold of an open neighbourhood of $\bar{\Omega}$ which intersects $b \Omega$ transversely such that $V \cap \Omega$ is biholomorphically equivalent to $\Delta$. Then $V \cap b \Omega$ is a 
simple closed curve which bounds $D$ in $V$ and which we denote by $b D$. Clearly $D=\varphi(\Delta)$ where $\varphi: \bar{\Delta} \rightarrow \mathbb{C}^{N}$ is a one to one continuous map which is holomorphic and regular on $\Delta$. We say that $\varphi$ parametrizes $D$. Let $D_{0}$ be a transversely embedded analytic disc and let $\varphi_{0}$ be a parametrization of $D_{0}$. A family $\mathcal{D}$ of such discs is called a neighbourhood of $D_{0}$ if there is an $\varepsilon>0$ such that $\mathcal{D}$ contains each transversely embedded analytic disc $D$ which can be parametrized by a map $\varphi_{D}$ satisfying $\left|\varphi_{D}(\zeta)-\varphi_{0}(\zeta)\right|<\varepsilon(\zeta \in \Delta)$.

As we shall see, the formulation of our principal result depends in an essential way on the linear structure of $\mathbb{C}^{N}$ so that it has no obvious analogue in the setting of domains in complex manifolds. However, in the final part of the paper we deduce from the principal result certain consequences that do hold on domains in Stein manifolds.

In formulating our main results it is convenient to use the notation that $\mathbb{C}^{1 ; 0}[d z]$ denotes the space of all $(1,0)$-forms on $\mathbb{C}^{N}$ with constant coefficients and $\mathbb{C}^{1 ; 1}[d z]$ the space of all $(1,0)$-forms with coefficients that are polynomials of degree not more than one. These spaces are invariant under the action of the group of affine automorphisms of $\mathbb{C}^{N}$ but under the action of no larger subgroup of the group $\operatorname{Aut}\left(\mathbb{C}^{N}\right)$.

The following is the principal result of the paper:

Theorem 1.1. Let $\Omega \subset \mathbb{C}^{N}$ be a bounded domain with boundary of class $\mathcal{C}^{2}$. Let $D_{0} \subset \Omega$ be a transversely embedded analytic disc and let $f$ be a continuous function in a neighbourhood of $b D_{0}$ in $b \Omega$. Let $w_{0}$ be a point of $D_{0}, z_{0}$ a point of $b D_{0}$. Assume that

$$
\int_{b D} f \omega=0
$$

for every $\omega \in \mathbb{C}^{1 ; 1}[d z]$ and for every transversely embedded analytic disc $D$ belonging to a neighbourhood $\mathcal{D}$ of $D_{0}$ such that $w_{0} \in D$. If $\Omega$ is strictly pseudoconvex at $z_{0}$ then the function $f$ is a CR function in a neighbourhood of $z_{0}$. If $\Omega$ is strictly convex at $z_{0}$, it suffices to assume the vanishing of the integrals (1.1) only when $\omega \in \mathbb{C}^{1 ; 0}[d z]$.

In the following $N$ is a fixed positive integer at least two. By $\mathbb{B}$ we denote the open unit ball in $\mathbb{C}^{N}$ or in $\mathbb{R}^{n}$, depending on the context. Similarly, $\mathbb{B}(z, r)$ is the ball of radius $r$ centered at the point $z$.

We describe the idea of the proof in the special case when $\Omega \subset \mathbb{C}^{2}$ is a convex domain and $D_{0}=\Lambda_{0} \cap b \Omega$ where $\Lambda_{0}$ is a complex line which meets $\Omega$. Let $L_{0}$ be the complex line tangent to $b \Omega$ at $z_{0}$. To prove that $f$ is a CR function in a neighbourhood of $z_{0}$ it is, by [G1S, Th. 3.2.1], enough to prove that

$$
\int_{L \cap b \Omega} f \omega=0
$$


for all $\omega \in \mathbb{C}^{1 ; 0}[d z]$ and for all complex lines $L$ which meet $\Omega$ and belong to a neighbourhood $\mathcal{L}$ of $L_{0}$ in the space of complex lines. Fix such a line $L$ and let $z \in L \cap b \Omega$. By the strict convexity of $\Omega$ at $z_{0}, z$ is close to $z_{0}$ provided that $\mathcal{L}$ is sufficiently small. Let $\Lambda$ be the complex line passing through $w_{0}$ and $z$. If $\mathcal{L}$ is sufficiently small then $\Lambda \cap \Omega$ belongs to $\mathcal{D}$. For convenience assume that $z=0$. Let $\ell$ and $h$ be linear functions on $\mathbb{C}^{2}$ such that $L=\left\{z \in \mathbb{C}^{2}: \ell(z)=0\right\}, \Lambda=\left\{z \in \mathbb{C}^{2}: h(z)=0\right\}$. We show that there are $\alpha \in \mathbb{R}$ and $\tau>0$ such that if $V_{t}=\left\{z \in \mathbb{C}^{2}: \ell(z) h(z)=t^{2} e^{i \alpha}\right\}$, $0<t<\tau$, and if $E$ is a unitary map such that $|E-I|<\tau$ then $E\left(V_{t}\right) \cap \Omega$ is a transversely embedded analytic disc which belongs to $\mathcal{D}$ provided that $\mathcal{L}$ and $\tau$ are small enough. For each $t, 0<t<\tau$, we choose $E_{t}$ in such a way that $w_{0} \in E_{t}\left(V_{t}\right)$ and that $E_{t} \rightarrow I$ as $t \rightarrow 0$. We show that if $t \rightarrow 0$ then $E_{t}\left(V_{t}\right) \cap b \Omega$ converges to $[\Lambda \cap b \Omega] \cup[L \cap b \Omega]$ in such a way that

$$
\int_{E_{t}\left(V_{t}\right) \cap b \Omega} f \omega \rightarrow \int_{\Lambda \cap b \Omega} f \omega+\int_{L \cap b \Omega} f \omega
$$

for each smooth 1 -form $\omega$ on $\mathbb{C}^{2}$. Now, for each $\omega \in \mathbb{C}^{1 ; 0}[d z]$ the terms on the left and the first term on the right vanish by the assumption so (1.2) holds.

In Section 2 we analyze carefully the intersections of the varieties $V_{t}=$ $\left\{(z, w) \in \mathbb{C}^{2}: z w=t^{2}, t>0\right\}$ with certain smooth perturbations of the real hyperplane $\left\{(z, w) \in \mathbb{C}^{2}: \operatorname{Re}(z+w)=0\right\}$. In Section 3 we use the results of this analysis to show the existence of $\alpha$ and $\tau$ with the properties above and then prove the theorem.

\section{Intersections in $\mathbb{C}^{2}$.}

To begin with, we study how certain small smooth perturbations of the real hyperplane

$$
H=\left\{(z, w) \in \mathbb{C}^{2}: \operatorname{Re}(z+w)=0\right\}
$$

intersect the variety $V_{1}=\left\{(z, w) \in \mathbb{C}^{2}: z w=1\right\}$.

The first step in this direction is to compute explicitly the intersection $V_{1} \cap H$ and to show that this intersection is transverse.

The transversality assertion goes as follows. Denote by $\Lambda$ the (unique) complex line in $H$ that passes through the origin. Thus, $\Lambda=\left\{(z, w) \in \mathbb{C}^{2}\right.$ : $w=-z\}$. At a point $\left(z, \frac{1}{z}\right)$ of $V_{1}$, the tangent space to $V_{1}$ is the complex line $\left\{\zeta\left(1,-1 / z^{2}\right): \zeta \in \mathbb{C}\right\}$. This coincides with $\Lambda$ if and only if $z^{2}=1$, i.e., if and only if $z= \pm 1$. It follows that $V_{1}$ meets the real hyperplane $H$ transversely.

The determination of $V_{1} \cap H$ uses the identification of $\mathbb{C}^{2}$ with $\mathbb{R}^{4}$ obtained by setting $z=x+i y$ and $w=u+i v$. The point $(z, w)$ lies in $V_{1} \cap H$ if and only if $x+u=0, x u-y v=1$, and $u y+x v=0$. These equations imply that $u=0, x=0$, and $y v=-1$. Thus

$$
V_{1} \cap H=\{(x+i y, u+i v): x=u=0, y v=-1\},
$$


a hyperbola contained in the (real) $(y, v)$-plane in $\mathbb{C}^{2}$. When studying the intersection of $V_{1}$ with perturbations of $H$, it is useful to view this hyperbola $y v=-1$ as the union of two graphs in a new coordinate system with the lines $y= \pm v$ as the coordinate axes. For this purpose introduce new coordinates in $\mathbb{R}^{4}$ by means of the real orthogonal transformation

$$
X=\frac{-x+u}{\sqrt{2}}, Y=\frac{-y+v}{\sqrt{2}}, U=\frac{x+u}{\sqrt{2}}, V=\frac{y+v}{\sqrt{2}} .
$$

The inverse of this is the orthogonal transformation given by

$$
x=\frac{-X+U}{\sqrt{2}}, y=\frac{-Y+V}{\sqrt{2}}, u=\frac{X+U}{\sqrt{2}}, v=\frac{Y+V}{\sqrt{2}} .
$$

With respect to the $(X, Y, U, V)$-coordinate system, the equation of $H$ is $U=0$, and

$$
V_{1} \cap H=\left\{\left(0, \sqrt{V^{2}+2}, 0, V\right): V \in \mathbb{R}\right\} \cup\left\{\left(0,-\sqrt{V^{2}+2}, 0, V\right): V \in \mathbb{R}\right\} .
$$

The perturbations of $H$ that we need to consider are graphs $\operatorname{Gr}(\varphi)$ of the form

$$
\operatorname{Gr}(\varphi)=\{(X, Y, U, V): U=\varphi(X, Y, V): X, Y, V \in \mathbb{R}\}
$$

where $\varphi$ is a function of class $\mathcal{C}^{1}$ on $\mathbb{R}^{3}$ such that

$$
|\varphi(p)|<\eta\left(p \in \mathbb{R}^{3},|p|<\rho\right) \text { and }|(D \varphi)(p)|<\eta\left(p \in \mathbb{R}^{3}\right)
$$

with suitably chosen $\rho$ and $\eta$. Note that in (2.1) the condition $|\varphi(p)|<\eta$ is assumed to hold only for $p \in \mathbb{R}^{3}$ in a fixed neighborhood of the origin; it need not hold on large subsets of $\mathbb{R}^{3}$. Nevertheless, it turns out that if $\eta$ is small enough, then $\operatorname{Gr}(\varphi) \cap H$ is similar to $V_{1} \cap H$ and, in particular, it is a union of two graphs:

Lemma 2.1. There are $\eta>0, \rho<\infty$, and $M<\infty$ such that whenever $\varphi$ is a function of class $\mathcal{C}^{1}$ on $\mathbb{R}^{3}$ that satisfies (2.1), then

(a) $\operatorname{Gr}(\varphi)$ is transverse to $V_{1}$, whence $\operatorname{Gr}(\varphi) \cap V_{1}$ is a closed submanifold of $\mathbb{R}^{4}$ of class $\mathcal{C}^{1}$,

(b) $\operatorname{Gr}(\varphi) \cap V_{1}=\Lambda_{1} \cup \Lambda_{2}$ where $\Lambda_{1}$ and $\Lambda_{2}$ are disjoint curves of class $\mathcal{C}^{1}$ each of which is of the form

$$
\Lambda_{j}=\left\{\left(X_{j}(V), Y_{j}(V), U_{j}(V), V\right): V \in \mathbb{R}\right\}
$$

where $X_{j}, Y_{j}$, and $U_{j}$ are $\mathcal{C}^{1}$ functions on $\mathbb{R}$ the first derivatives of which are bounded by $M$, and

(c) $Y_{1}(V)>0$ and $Y_{2}(V)<0 \quad(V \in \mathbb{R})$.

Remark 1. If $\varphi \equiv 0$, then $\operatorname{Gr}(\varphi)=H, U_{j}=X_{j}=0$, and $Y_{1}, Y_{2}$ are the functions $V \mapsto \pm \sqrt{V^{2}+2}$. 
Remark 2. Having proved (a), to prove (b) it is enough to prove that there is a $\beta>0$ such that for each $s \in \mathbb{R}, \operatorname{Gr}(\varphi) \cap V_{1}$ intersects the hyperplane

$$
E_{s}=\{(X, Y, U, s): X, Y, U \in \mathbb{R}\}
$$

at precisely two points and, moreover, that if $p \in \operatorname{Gr}(\varphi) \cap V_{1} \cap E_{s}$, then the angle between the hyperplane $E_{s}$ and the (tangent line to) the curve $\operatorname{Gr}(\varphi) \cap V_{1}$ at $p$ is bounded below by $\beta$.

The proof of Lemma 2.1 requires some preliminaries.

Denote by $L_{1}$ and $L_{2}$ the $z$ - and $w$-axes in $\mathbb{C}^{2}$, respectively. Far from the origin, $V_{1}$ is a slight perturbation of $L_{1} \cup L_{2}$, so to understand $\operatorname{Gr}(\varphi) \cap V_{1}$ far from the origin, we first understand how $\operatorname{Gr}(\varphi)$ intersects $L$, a small perturbation of $L_{1}$ or $L_{2}$.

Lemma 2.2. There are $\delta, \beta>0$ such that if $\varphi$ is a $\mathcal{C}^{1}$ function on $\mathbb{R}^{3}$ with

$$
|(D \varphi)(p)|<\delta \quad\left(p \in \mathbb{R}^{3}\right)
$$

and if $f, g$ are functions of class $\mathcal{C}^{1}$ on $\mathbb{R}^{2}$ that satisfy

$$
\|f\|_{\mathcal{C}^{1}\left(\mathbb{R}^{2}\right)}<\delta, \quad\|g\|_{\mathcal{C}^{1}\left(\mathbb{R}^{2}\right)}<\delta
$$

then with $L=\{(x, y, f(x, y), g(x, y)): x, y \in \mathbb{R}\}$ we have that

(a) $\operatorname{Gr}(\varphi)$ is transverse to $L$,

(b) for each $s, \operatorname{Gr}(\varphi) \cap L \cap E_{s}$ consists of a single point, and

(c) if $p \in \operatorname{Gr}(\varphi) \cap E_{s}$, then the angle between $E_{s}$ and $\operatorname{Gr}(\varphi) \cap L$ at $p$ is bounded below by $\beta$.

Proof. The $z$-axis $L_{1}=\{(x, y, 0,0): x, y \in \mathbb{R}\}$ intersects $H$ transversely, and the line $L_{1} \cap H$ intersects $E_{s}$ transversely at an angle that does not depend on $s$. Further, (2.3) implies that at each point $p$ of $L$, the tangent space $T_{p} L$ is arbitrarily close to $L_{1}$, uniformly with respect to $p \in L$ provided that $\delta$ is small enough, and (2.2) implies that each point $p \in \operatorname{Gr}(\varphi), T_{p} G r(\varphi)$ is arbitrarily close to $H$, uniformly with respect to $p$, provided that $\delta$ is small enough. This shows that $\operatorname{Gr}(\varphi)$ is transverse to $L$ if $\delta$ is small enough, which proves (a). It also shows that the tangent line to $L \cap G r(\varphi)$ at a point $p$ is arbitrarily close to $L_{1} \cap H$ when $\delta$ is small enough, which proves that there is a $\beta>0$ satisfying (c) when $\delta$ is small enough.

If $s \in \mathbb{R}$, then $L \cap E_{s}=\{(x, y, f(x, y), g(x, y)): y+g(x, y)=s \sqrt{2}\}$. If $\delta$ is small enough, then a simple one-variable argument shows that given $s, x \in$ $\mathbb{R}$, there is a unique $y=y(x, s)=s \sqrt{2}+\psi(x, s)$ such that $y+g(x, y)=s \sqrt{2}$, and, moreover, that $\psi(x, s)$ is arbitrarily small, uniformly with respect to $x, s \in \mathbb{R}$ when $\delta$ is sufficiently small. By the implicit mapping theorem $\psi$ is of class $\mathcal{C}^{1}$. By differentiating the equality $\psi(x, s)+g(x, s \sqrt{2}+\psi(x, s))=0$, we see that $\frac{\partial \psi}{\partial x}$ and $\frac{\partial \psi}{\partial s}$ are arbitrarily small, uniformly in $x, s \in \mathbb{R}$ when $\delta$ is small. If we put $t=-x / \sqrt{2}$ and pass to $X, Y, U, V$-coordinates, we can 
write $L \cap E_{s}=F_{s}(\mathbb{R})$ where $F_{s}(t)=(t+A(t, s),-s+B(t, s),-t+A(t, s), s)$ with $\|A\|_{\mathcal{C}^{1}},\|B\|_{\mathcal{C}^{1}}$ arbitrarily small provided that $\delta$ is small enough. The set $E_{s} \cap \operatorname{Gr}(\varphi)$ has two (connected) components $\Omega_{s}^{-}=\{(X, Y, U, s): U<$ $\varphi(X, Y, s)\}$ and $\Omega_{s}^{+}=\{(X, Y, U, s): U>\varphi(X, Y, s)\}$. If $p \in G r(\varphi) \cap E_{s}$, let $n_{p}$ be the unit normal vector to $G r(\varphi) \cap E_{s}$ at $p$ that points in the direction of $\Omega_{s}^{+}$. By $(2.2), n_{p}$ is arbitrarily close to $(0,0,1,0)$, uniformly with respect to $p$ if $\delta$ is small enough. Further, for sufficiently small $\delta, \dot{F}(t) /|\dot{F}(t)|$ is arbitrarily close to $\left(\frac{1}{\sqrt{2}}, 0,-\frac{1}{\sqrt{2}}, 0\right)$, uniformly with respect to $s$ and $t$. Thus, granted that $\delta$ is small enough, if $\langle$,$\rangle denotes the real inner product on \mathbb{R}^{4}$, then

$$
\left\langle\frac{\dot{F}_{s}(t)}{\left|\dot{F}_{s}(t)\right|}, n_{p}\right\rangle<0 \quad\left(t \in \mathbb{R}, p \in G r(\varphi) \cap E_{s}\right),
$$

which shows that only way that $F_{s}(t)$ can meet $\operatorname{Gr}(\varphi)$ as $t$ increases is when it passes from $\Omega_{s}^{+}$to $\Omega_{s}^{-}$, which implies that $\operatorname{Gr}(\varphi) \cap E_{s} \cap L$ contains at most one point.

To see that $\operatorname{Gr}(\varphi) \cap E_{s} \cap L \neq \emptyset$ we must show that $\varphi(t+A(t, s),-s+$ $B(t, s), s)=-t+A(t, s)$ for at least one $t$. For small $\delta$ this follows from the one-variable fact that if $p, q$ are two functions of class $\mathcal{C}^{1}$ on $\mathbb{R}$ such that $p^{\prime}(t)<\mu<\nu<q^{\prime}(t), t \in \mathbb{R}$, for two constants $\mu$ and $\nu$, then their graphs intersect.

Lemma 2.3. If $R>0$, then the $\delta$ of Lemma 2.2 can be chosen so small that if (2.2) and (2.3) are satisfied, and if, in addition, $\varphi$ satisfies

$$
|\varphi(X, Y, V)|<\delta \quad(|X|,|Y|,|V|<2 R)
$$

then $|s| \leq R$ and $(X, Y, U, s) \in G r(\varphi) \cap L \cap E_{s}$ imply that $|X|<R$ and $|Y|<2 R$.

Proof. Choose $\omega \in(0, \min \{1, R\})$ such that $2 \omega /(1-\omega)<R-\omega$. Let $A, B$ be as in the proof of Lemma 2.2. Choose $\delta<\omega$ so small that Lemma 2.2 holds, that $|A(t, s)|<\omega,|B(t, s)|<\omega(t, s \in \mathbb{R})$, and that $\varphi$ satisfies

$$
\left|\frac{d}{d t} \varphi(t+A(t, s),-s+B(t, s), s)\right|<\omega \quad(t, s \in \mathbb{R}) .
$$

Assume also that $\varphi$ satisfies (2.4). Recall that $\operatorname{Gr}(\varphi) \cap L \cap E_{s}=\{(\lambda+$ $A(\lambda, s),-s+B(\lambda, s),-\lambda+A(\lambda, s), s)\}$ where $-\lambda+A(\lambda, s)=\varphi(\lambda+A(\lambda, s),-s$ $+B(\lambda, s), s)$. It follows that

$$
\begin{aligned}
-\lambda & +A(\lambda, s) \\
& =\int_{0}^{\lambda} \frac{d}{d t} \varphi(t+A(t, s),-s+B(t, s), s) d t+\varphi(A(0, s),-s+B(0, s), s) .
\end{aligned}
$$

Since $|A(0, s)|,|B(0, s)|<R$, it follows by (2.4) that $|s| \leq R$ implies that

$$
|\varphi(A(0, s),-s+B(0, s), s)|<\delta<\omega
$$


whence (2.5) implies that $|\lambda|<2 \omega /(1-\omega)<R-\omega$ so $|-\lambda+A(\lambda, s)|<R$. Clearly $|-s+B(t, s)|<R+R=2 R$. This completes the proof.

To prove Lemma 2.1, we also need the following quantitative form of the inverse function theorem:

Lemma 2.4. Let $\mathcal{U}$ be an open subset of $\mathbb{R}^{n}$ that contains the origin, and let $F: \mathcal{U} \rightarrow R^{n}$ be a map of class $\mathcal{C}^{1}$ such that $F(0)=0$ and $(D F)(0)$ is nonsingular. If $r>0$ is so small that

$$
|(D F)(x)-(D F)(0)|<\frac{1}{8\left|(D F)(0)^{-1}\right|} \quad(|x|<r),
$$

then given a $\mathcal{C}^{1}$ map $\varphi: \mathcal{U} \rightarrow \mathbb{R}^{n}$ such that

$$
|(D \varphi)(x)|<\frac{1}{16\left|(D F)(0)^{-1}\right|} \quad(|x|<r)
$$

and

$$
|\varphi(0)|<\frac{r}{4\left|(D F)(0)^{-1}\right|},
$$

there is a unique $x$ with $|x|<r$ such that $F(x)+\varphi(x)=0$.

Remark 3. The assumptions imply that if $|x|<r$, then $D(F+\varphi)(x)$ is nonsingular. Indeed, if $|x|<r$, then $D F(0)^{-1} D(F+\varphi)(x)$ is invertible:

$$
\begin{aligned}
& \left|D F(0)^{-1} D(F+\varphi)(x)-I\right| \\
& =\left|D F(0)^{-1}\{D(F+\varphi)(x)-D F(0)\}\right| \\
& \leq\left|D F(0)^{-1}\right|\{|D F(x)-D F(0)|+|D \varphi(x)|\} \\
& \leq\left|D F(0)^{-1}\right|\left\{\frac{1}{8} \frac{1}{\left|D F(0)^{-1}\right|}+\frac{1}{16} \frac{1}{\left|D F(0)^{-1}\right|}\right\}=3 / 16<1 .
\end{aligned}
$$

The proof of Lemma 2.4 is in the Appendix to this section.

Proof of Lemma 2.1. For each $t>0$, set $D_{t}=\{(X, Y, U, V):|V|<t\}$, and let $L_{1}, L_{2}$ be the coordinate axes in $\mathbb{C}^{2}$. Sufficiently far away from the origin, $V_{1}$ is an arbitrarily small perturbation of $L_{1} \cup L_{2}$, so by Lemmas 2.2 and 2.3 , there are $R, 2<R<\infty, \delta>0$, and $\gamma>0$ such that if $\varphi$ satisfies (2.2) and (2.4), then:

(i) $\operatorname{Gr}(\varphi)$ is transverse to $V_{1}$ at each point of $\mathbb{C}^{2} \backslash D_{R}$,

(ii) for each $s,|s| \geq R$, the set $\operatorname{Gr}(\varphi) \cap V_{1} \cap E_{s}$ consists of precisely two points and at each of these points the angle between (the tangent line to) $\operatorname{Gr}(\varphi) \cap V_{1}$ and $E_{s}$ is at least $\gamma$, and

(iii) if $|s| \leq R$ and if $(X, Y, U, s) \in G r(\varphi) \cap V_{1}$, then $|X|<R$ and $|Y|<2 R$.

Let the $\rho$ of condition (2.1) satisfy $\rho>8 R$. To complete the proof, it suffices to show that there are $\eta, 0<\eta<\delta$, and $\gamma^{\prime}>0$ such that if $\varphi$ satisfies (2.1), then $G(\varphi)$ is transverse to $V_{1}$ at each point of $\bar{D}_{R}$ and that 
for each $s,|s|<R$, the set $\{|X|<R,|Y|<2 R\} \cap V_{1} \cap G r(\varphi) \cap E_{s}$ consists of precisely two points at each of which the angle between (the tangent line to) $V_{1} \cap \operatorname{Gr}(\varphi)$ and $E_{s}$ is at least $\gamma^{\prime}$.

The equations of $V_{1}$ in the $(X, Y, U, V)$-coordinates are $-X^{2}+Y^{2}+U^{2}-$ $V^{2}=2$ and $X Y-U V=0$, so given $s \in \mathbb{R}$, we find $E_{s} \cap V_{1} \cap \operatorname{Gr}(\varphi)$ by solving $\tilde{F}(X, Y, U, V)=(0,0,0, s)$ where

$\tilde{F}(X, Y, U, V)=\left(-X^{2}+Y^{2}+U^{2}-V^{2}-2, X Y-U V, U-\varphi(X, Y, V), V\right)$.

Let

$$
F(X, Y, U, V)=\left(-X^{2}+Y^{2}+U^{2}-V^{2}-2, X Y-U V, U, V\right) .
$$

Then $F^{-1}(0,0,0, s)=V_{1} \cap H \cap E_{s}=\left\{\left(0, \sqrt{2+s^{2}}, 0, s\right),\left(0,-\sqrt{2+s^{2}}, 0, s\right)\right\}$. We have $\operatorname{det}\left[D F\left(0, \pm \sqrt{2+s^{2}}, 0, s\right)\right]=-2\left(2+s^{2}\right)(s \in \mathbb{R})$, so $(D F)(p)$ is invertible at each point of $V_{1} \cap H$. Clearly $(D F)(p)$ and $(D F)(p)^{-1}$ depend continuously on $p=\left(0, \pm \sqrt{2+s^{2}}, 0, s\right)$ on each branch of $V_{1} \cap H$, i.e., they depend continuously on $s$. By compactness Lemma 2.4 now implies that

(A) there are $r, 0<r<\sqrt{R^{2}+2}-R$ and $\beta>0$ such that whenever $p=\left(0, \pm \sqrt{s^{2}+2}, 0, s\right)$ with $|s| \leq R$ and $\varphi$ satisfies $|\varphi|<\beta$ and $|D \varphi|<\beta$ on $(p+r \mathbb{B}) \cap H$, then $\operatorname{Gr}(\varphi) \cap \bar{V}_{1} \cap E_{s} \cap(p+r \mathbb{B})$ consists of precisely one point, at which $D \tilde{F}$ is nonsingular.

The nonsingularity of $D \tilde{F}$ at $p$ means that $\operatorname{Gr}(\varphi)$ is transverse to $V_{1}$ at $p$ and that the angle between $\operatorname{Gr}(\varphi) \cap V_{1}$ and $E_{s}$ at $p$ is positive. Since this angle depends continuously on $s$, it follows that for $|s| \leq R$ it is bounded below by a positive constant.

The equation of $V_{1} \cap H$ is $X=U=0, Y= \pm \sqrt{2+V^{2}}$. Since $R>2$ and $r<\sqrt{R^{2}+2}-R$, it follows that $\left|X^{\prime}\right|<R$ and $\left|Y^{\prime}\right|<2 R$ whenever $|V| \leq$ $R, p=(X, Y, 0, V) \in V_{1} \cap H$, and $p^{\prime}=\left(X^{\prime}, Y^{\prime}, U^{\prime}, V\right)$ satisfies $\left|p^{\prime}-p\right|<r$. Let $\mathcal{U}=\left[\bar{D}_{R} \cap V_{1} \cap H\right]+r \mathbb{B}$. By compactness there is some $\eta, 0<\eta<\min \{\delta, \beta\}$, such that $\bar{D}_{R} \cap V_{1} \cap\{(X, Y, U, V):|X|<R,|Y|<2 R,|U|<\eta\} \subset \mathcal{U}$. Since $\rho>8 R$, it follows that if $\varphi$ satisfies (2.1) then $|\varphi|<\beta$ and $|D \varphi|<\beta$ on $\mathcal{U} \cap H$, which, by $(\mathbf{A})$, implies that if $|s| \leq R$, then the only points $(X, Y, U, V)$ in $E_{s} \cap V_{1} \cap G r(\varphi)$ that satisfy $|X|<R,|Y|<2 R$ are the two described in $(\mathbf{A})$.

It remains only to prove assertion (c). For this, note that $V_{1} \backslash \mathbb{B}(0, r)$ is an arbitrarily small perturbation of $\left(L_{1} \cup L_{2}\right) \backslash \mathbb{B}(0, r)$ where the $L_{i}$ are the coordinate axes, provided that $r$ is large enough. In particular, if $\rho$ in Lemma 2.1 is large enough and $\eta>0$ small enough then for, say, $|V|>\rho / 4$, the tangent line to $\Lambda_{i}$ is arbitrarily close to either $L_{1} \cap H=\{X=U=V=$ $0\}=\{X=U=0, V=-Y\}$ or to $L_{2} \cap H=\{X=U=Y=0\}=\{X=$ $U=0, V=Y\}$. In particular, there are disjoint neighborhoods $\mathcal{W}_{1}$ of $L_{1} \cap H$ and $\mathcal{W}_{2}$ of $L_{2} \cap H$ in the space of real lines passing through the origin such that the tangent line to $\Lambda_{i}$ for $|V|>\rho / 4$ is either in $\mathcal{W}_{1}$ or in $\mathcal{W}_{2}$. With 
no loss of generality, assume that if $\left\{X=p_{1} V, Y=p_{2} V, U=p_{3} V: V \in \mathbb{R}\right\}$ belongs to $\mathcal{W}_{1}$, then $p_{2}>\frac{1}{2}$ and if it belongs to $\mathcal{W}_{2}$, then $p_{2}<-\frac{1}{2}$. On the other hand, provided that $\eta$ is small enough, $\left\{V_{1} \cap \operatorname{Gr}(\varphi):|V|<\rho / 2\right\}$ is an arbitrarily small perturbation of $\left\{V_{1} \cap H:|V|<\rho / 2\right\}=\{X=U=$ $\left.0, Y=-\sqrt{2+V^{2}}\right\} \cup\left\{X=U=0, Y=\sqrt{2+V^{2}}\right\}$. It follows that one of the functions $Y_{i}$, say $Y_{1}$, is positive on $|V| \leq \rho / 2$ and the other, $Y_{2}$, is negative there. By the preceding discussion $Y_{1}^{\prime}(\rho / 2)>\frac{1}{2}$, and $Y_{1}^{\prime}(\rho / 2)<-\frac{1}{2}$, and $Y_{2}^{\prime}(\rho / 2)<-\frac{1}{2}$, and $Y_{2}^{\prime}(\rho / 2)>\frac{1}{2}$ provided that $\eta$ is small enough. Since for $|V| \geq \rho / 4$, either $Y_{i}^{\prime}(V)>\frac{1}{2}$ or $Y_{i}^{\prime}(V)<-\frac{1}{2}$, it follows, by the continuity of $Y_{i}^{\prime}$ that $Y_{1}$ decreases on $(-\infty,-\rho / 4)$ and increases on $(\rho / 4, \infty)$ and that $Y_{2}$ increases on $(-\infty, \rho / 4)$ and decreases on $(\rho / 4, \infty)$. This establishes (c) and completes the proof of Lemma 2.1.

We now want to understand how a surface $S$ passing through the origin and almost tangent to $H=\{(z, w): \operatorname{Re}(z+w)=0\}$ intersects the varieties $V_{t}=\left\{(z, w): z w=t^{2}\right\}$ for small $t>0$ in a neighborhood of the origin. To do this, we consider a $\mathcal{C}^{1}$ function $\psi$ on $\mathbb{R}^{3}$ that satisfies $\psi(0)=0$ and consider how $S=G r(\psi)$ intersects $V_{t}$.

The main fact we use is the homogeneity condition that $V_{t}=t V_{1}$ when $t>0$. This implies that $V_{t} \cap G r(\psi)=t\left(V_{1} \cap \frac{1}{t} G r(\psi)\right)$. Notice that $\frac{1}{t} G r(\psi)=$ $\operatorname{Gr}(\varphi)$ if $\varphi(X, Y, V)=\frac{1}{t} \psi(t X, t Y, t V)$. Also $(D \varphi)(X, Y, V)=(D \psi)(t X, t Y$, $t V)$, so $|(D \varphi)(p)|<\tau$ for all $p \in \mathbb{R}^{3}$ if and only $|(D \psi)(p)|<\tau$ for all $p \in \mathbb{R}^{3}$.

If $\eta, \rho$, and $M$ are as in Lemma 2.1, then the conditions $(2.1)$ for $\varphi(p)=$ $\frac{1}{t} \psi(t p)$ are

$$
\left|\frac{1}{t} \psi(t p)\right|<\eta \quad(|p|<\rho) \text { and }|(D \psi)(p)|<\eta \quad\left(p \in \mathbb{R}^{3}\right) .
$$

Lemma 2.5. There are $\tau>0$ and $M<\infty$ such that if the smooth real function $\psi$ on $\mathbb{R}^{3}$ satisfies

$$
\psi(0)=0 \text { and }|(D \psi)(p)|<\tau \quad\left(p \in \mathbb{R}^{3}\right),
$$

then for every $t>0$

(a) $\operatorname{Gr}(\psi)$ is transverse to $V_{t}$, and

(b) $\operatorname{Gr}(\psi) \cap V_{t}=\Lambda_{1} \cup \Lambda_{2}$ where $\Lambda_{1}$ and $\Lambda_{2}$ are $\mathcal{C}^{1}$ curves of the form

$$
\Lambda_{j}=\left\{\left(X_{j}(V), Y_{j}(V), U_{j}(V), V\right): V \in \mathbb{R}\right\}
$$

where the functions $X_{j}, Y_{j}$, and $U_{j}$ are of class $\mathcal{C}^{1}$ on $\mathbb{R}$ and where the first derivative of each is bounded uniformly by $M$, and

(c) $Y_{1}(V)>0$ and $Y_{2}(V)<0 \quad(V \in \mathbb{R})$.

Moreover, given $\epsilon>0$, there is $\delta>0$ such that if

$$
W=\{(X, Y, U, V):|V|<\delta\},
$$


then for all $t>0$

$$
\text { length }\left(W \cap V_{t} \cap G r(\psi)\right)<\epsilon \text {. }
$$

Proof. Let $\eta, \rho$ and $M$ be as in Lemma 2.1. This lemma and the preceding discussion imply that to prove (a)-(c) it suffices to show that $\tau>0$ in (2.10) can be chosen so small that (2.10) implies (2.9) for every $t>0$. Let $\tau=\min \{\eta, \eta / \rho\}$, and assume that $\psi$ satisfies (2.10). The second inequality in (2.9) is obviously satisfied. To prove the first inequality, let $|p|<\rho$. Then (2.10) implies that

$$
\left|\frac{1}{t} \psi(t p)\right|=\left|\frac{1}{t} \int_{0}^{t}(D \psi)(\lambda p) \cdot p d \lambda\right| \leq \frac{1}{t} \tau|p| t<\frac{\eta}{\rho} \rho=\eta .
$$

Finally, if $\delta>0$ is so small that $\delta \sqrt{3 M^{2}+1}<\epsilon$, and if $W$ is given by (2.11) then

$$
\begin{aligned}
\operatorname{length}\left(W \cap V_{t} \cap G r(\varphi)\right) & =\sum_{j=1}^{2} \int_{-\delta}^{\delta} \sqrt{X_{j}^{\prime}(t)^{2}+Y_{j}^{\prime}(t)^{2}+U_{j}^{\prime}(t)^{2}+1} d t \\
& \leq 4 \delta \sqrt{3 M^{2}+1}<\epsilon .
\end{aligned}
$$

This completes the proof.

\section{Appendix to Section 2.}

The proof of Lemma 2.4 depends on the following standard result:

Lemma 2.A.1. Let $\mathcal{U}$ be a neighborhood of $0 \in \mathbb{R}^{n}$, and let $F: \mathcal{U} \rightarrow \mathbb{R}^{n}$ be a $\mathcal{C}^{1}$ map with $F(0)=0$ and $(D F)(0)=I$. If $r>0$ is so small that $|(D F)(0)(x)-(D F)(0)|<\frac{1}{2}$ when $|x|<r$, then for each $y,|y|<\frac{r}{2}$, there is precisely one $x,|x|<r$, such that $F(x)=y$.

Proof. Let $G(x)=F(x)-x$. Then $|(D G)(x)|<\frac{1}{2}$ when $|x|<r$. Thus, if $|x|,|y|<r$, then $|G(x)-G(y)|=\left|\int_{0}^{1}(D G)(x+t(y-x))(y-x) d t\right| \leq \frac{1}{2}|x-y|$, so $\frac{1}{2}|x-y| \geq|G(x)-G(y)|=|F(x)-F(y)-(x-y)| \geq|x-y|-|F(x)-F(y)|$, and $|F(x)-F(y)| \geq \frac{1}{2}|x-y|$, whence $F$ is one-to-one on $W=\{|x|<$ $r\}$. Let $|y|<\frac{r}{2}$ and set $x_{0}=0, x_{n}=y-G\left(x_{n-1}\right), n=1,2, \ldots$ Then $\left|x_{n+1}-x_{n}\right|=\left|G\left(x_{n}\right)-G\left(x_{n-1}\right)\right| \leq \frac{1}{2}\left|x_{n}-x_{n-1}\right|$. Note that $x_{n}, x_{n-1}$ do lie in $\mathcal{W}:\left|x_{1}\right|=|y|<\frac{r}{2}$, so $\left|x_{1}-x_{0}\right|<\frac{r}{2} \leq \frac{1}{2}\left|x_{2}-x_{1}\right| \leq \frac{1}{2}\left|x_{1}-x_{0}\right| \leq \frac{1}{2} \frac{r}{2}$, etc. Thus, $x_{n} \rightarrow x$, and $x=y-F(x)+x$, whence $y=F(x)$. This completes the proof of the Lemma.

Lemma 2.A.2. Let $\mathcal{U}$ be a neighborhood of the origin in $\mathbb{R}^{n}$, and let $F$ : $\mathcal{U} \rightarrow \mathbb{R}^{n}$ be a $\mathcal{C}^{1}$ map with $F(0)=0$ and $(D F)(0)$ nonsingular. If $r>0$ is so small that

$$
|(D F)(x)-(D F)(0)|<\frac{1}{2\left|(D F)(0)^{-1}\right|} \text { when }|x|<r
$$


then for each $z$ with $|z|<\frac{r}{2\left|(D F)(0)^{-1}\right|}$ there is a unique $x,|x|<r$, such that $F(x)=z$.

Proof. Consider the map $x \mapsto(D F)(0)^{-1} \circ F(x)=G(x)$. We have that $G(0)=0$ and that $|(D G)(x)-(D G)(0)|=\left|D F(0)^{-1}[(D F)(x)-(D F)(0)]\right| \leq$ $\left|(D F)(0)^{-1}\right| \cdot|(D F)(x)-(D F)(0)|<\frac{1}{2}$ when $|x|<r$. By Lemma 2.A.1, given $y,|y|<\frac{r}{2}$, there is a unique $x,|x|<r$, such that $G(x)=y$, i.e., $F(x)=(D F)(0)(y)$. Consequently, for each $z \in \frac{r}{2}(D F)(0)(\mathbb{B})$, there is a unique $x,|x|<r$, such that $F(x)=z$. As $\frac{1}{\left|(D F)(0)^{-1}\right|} \mathbb{B} \subset(D F)(0)(\mathbb{B})$ it follows that for each $z,|z|<\frac{r}{2} \frac{1}{\left|D F(0)^{-1}\right|}$ there is precisely one $x$ with $|x|<r$ such that $F(x)=z$. This completes the proof.

Proof of Lemma 2.4. Put $G(x)=F(x)+\varphi(x)-\varphi(0)$ so that $G(0)=0$, and $(D G)(x)=(D F)(x)+(D \varphi)(x)$. We have

$$
\begin{aligned}
(D G)(0)^{-1} & =[(D F)(0)+(D \varphi)(0)]^{-1} \\
& =(D F)(0)^{-1} \sum_{k=0}^{\infty}\left[-(D F)(0)^{-1} D \varphi(0)\right]^{k} .
\end{aligned}
$$

By $(2.7)\left|(D F)(0)^{-1} D \varphi(0)\right| \leq\left|(D F)(0)^{-1}\right||(D \varphi)(0)|<\frac{1}{2}$, so

$$
\left|(D G)(0)^{-1}\right| \leq 2|(D F)(0)|^{-1} .
$$

Further, if $|x|<r$, then by (2.7) and (2.8)

$$
\begin{aligned}
|(D G)(x)-(D G)(0)| & \leq|(D F)(x)-(D F)(0)|+|(D \varphi)(0)|+|(D \varphi)(x)| \\
& <\frac{1}{8} \frac{1}{\left|D F(0)^{-1}\right|}+\frac{1}{8} \frac{1}{\left|D F(0)^{-1}\right|} \\
& <\frac{1}{4} \frac{1}{\left|D F(0)^{-1}\right|} \leq \frac{1}{2\left|D G(0)^{-1}\right|}
\end{aligned}
$$

so by Lemma 2.A.2, for each $|z|<\frac{r}{2\left|D G(0)^{-1}\right|}$ there is a unique $x,|x|<r$, such that $G(x)=z$. In particular, by (2.8) and (2.12), this holds for $z=-\varphi(0)$. Accordingly, there is a unique $x,|x|<r$, such that $F(x)+\varphi(x)=0$. This completes the proof of Lemma 2.4.

\section{Proof of Theorem 1.1.}

We begin with two lemmas of a general character, which will be used in the main part of the proof of the theorem.

The proof of the first of these is quite short but is decidedly nontrivial, as it depends essentially on some complicated results in the theory of polynomial convexity. 
Lemma 3.1. If $\varphi: \bar{\Delta} \rightarrow \mathbb{C}^{N}$ is a continuous map that is holomorphic on $\Delta$, that is one-to-one on $\bar{\Delta}$ and that carries $b \Delta$ onto a rectifiable simple closed curve, then the set $X=\varphi(\bar{\Delta})$ is polynomially convex.

Proof. Let $\Sigma$ be the image of $b \Delta$ under $\varphi$, and denote by $\widehat{\Sigma}$ the polynomially convex hull of $\Sigma$. It is a result of Alexander [Al1] that $\widehat{\Sigma} \backslash \Sigma$ is a closed analytic subvariety of the domain $\mathbb{C}^{N} \backslash \Sigma$. Moreover, by [Al2], this variety is irreducible. As $\varphi(\Delta)$ is a closed analytic subvariety of $\mathbb{C}^{N} \backslash \Sigma$ that is contained in $\widehat{\Sigma}$, it follows that, as claimed, $X=\widehat{\Sigma}$.

Lemma 3.2. Let $P \subset \mathbb{C}^{2}$ be a bounded domain. Let $V_{t}=\{(z, w): z w=$ $\left.t^{2}\right\}, t>0$, and suppose that $V_{t} \cap P \neq \emptyset$ and that $V_{t} \cap b P=\Gamma$ is a simple closed curve. Then $V_{t} \cap P$ is biholomorphically equivalent to a disc.

Proof. The projection $\pi: \mathbb{C}^{2} \rightarrow \mathbb{C}$ given by $\pi(z, w)=z$ carries $V_{t}$ biholomorphically onto $\mathbb{C} \backslash\{0\}$. Let $Q$ be the bounded component of $\mathbb{C} \backslash \pi(\Gamma)$. Since $P$ is bounded, it follows that if $z \in \mathbb{C} \backslash \bar{Q}, z \neq 0$, then $\left(z, \frac{t^{2}}{z}\right) \in \mathbb{C}^{2} \backslash \bar{P}$. We show that $0 \notin Q$. Otherwise, there are points $z_{n} \in Q$ with $z_{n} \rightarrow 0$ such that $\left(z_{n}, \frac{t^{2}}{z_{n}}\right) \in P$ for all $n$, contradicting the boundedness of $P$. Thus, $0 \notin Q$, and $\left\{\left(z, \frac{t^{2}}{z}\right): z \in Q\right\}=P \cap V_{t}$. As $Q$ is biholomorphically equivalent to a disc, the same is true of $P \cap V_{t}$. This completes the proof.

We now begin the proof of Theorem 1.1 itself. Initially we work in $\mathbb{C}^{2}$. We shall deal first with the case that $\Omega$ is strictly convex at $z_{0}$.

By the assumption of convexity, there are a ball $\mathbb{B}\left(z_{o}, r\right)$ centered at $z_{0}$ and a function $\varrho$ on $\mathbb{B}\left(z_{0}, r\right)$ with nonvanishing gradient and positive definite Hessian such that $\mathbb{B}\left(z_{0}, r\right) \cap b \Omega=\left\{w \in \mathbb{B}\left(z_{0}, r\right): \varrho(w)=0\right\}$ and $\mathbb{B}\left(z_{0}, r\right) \cap \Omega=$ $\left\{w \in \mathbb{B}\left(z_{o}, r\right): \varrho(w)<0\right\}$. Denote by $\Lambda\left(z_{0}, b \Omega\right)$ the complex line passing through $z_{0}$ and tangent to $b \Omega$ at $z_{0}$. There is a neighborhood $\mathcal{L}$ of $\Lambda\left(z_{0}, b \Omega\right)$ in the space of all complex lines in $\mathbb{C}^{2}$ such that if $\Lambda \in \mathcal{L}$ meets $\mathbb{B}\left(z_{0}, r\right) \cap \Omega$, then $\Lambda$ meets $\mathbb{B}\left(z_{0}, r\right) \cap b \Omega$ transversely in a closed curve that bounds a convex domain in $\Lambda$. We shall show, after passing to a smaller $\mathcal{L}$ if necessary, that

$$
\int_{\Lambda \cap \mathbb{B}\left(z_{0}, r\right) \cap b \Omega} f \omega=0 \quad(\Lambda \in \mathcal{L})
$$

for all $\omega \in \mathbb{C}^{1 ; 0}[d z]$. By [GlS, Th. 3.2.1] this implies that $f$ is a CR-function in a neighborhood of $z_{0}$.

Given $\Lambda \in \mathcal{L}$ which meets $\mathbb{B}(0, r) \cap \Omega$ we shall show that the neighbourhood $\mathcal{D}$ contains transversely embedded analytic discs $D_{n}, n \in \mathbb{N}$, and $D$, such that as $n \rightarrow \infty, b D_{n}$ converges to $b D \cup\left[\Lambda \cap \mathbb{B}\left(z_{0}, r\right) \cap b \Omega\right]$ in the sense that for every continuous 1 -form $\gamma$ on $\mathbb{C}^{2}$,

$$
\lim _{n \rightarrow \infty} \int_{b D_{n}} \gamma=\int_{b D} \gamma+\int_{\Lambda \cap \mathbb{B}\left(z_{0}, r\right) \cap b \Omega} \gamma
$$


If $\omega \in \mathbb{C}^{1 ; 0}[d z]$, then by hypothesis $\int_{b D_{n}} f \omega=0,(n \in \mathbb{N})$, and $\int_{b D} f \omega=0$. Applying (3.2) with $\gamma=f \omega$ gives (3.1).

To construct such sequences, first consider the following special case. We again use the notation that $V_{t}=\left\{(z, w) \in \mathbb{C}^{2}: z w=t^{2}\right\}(t>0)$.

Lemma 3.3. Let $\Omega \subset \mathbb{C}^{2}$ be a bounded domain with boundary of class $\mathcal{C}^{2}$. Let $0 \in b \Omega$, and let $\operatorname{Re}(z+w)=0$ be the tangent plane to $b \Omega$ at 0 . Denote by $L_{1}$ and $L_{2}$ the coordinate axes of $\mathbb{C}^{2}$, which we assume to intersect $b \Omega$ transversely and in such a way that $L_{j} \cap b \Omega=\Lambda_{j}$ is a simple closed curve that bounds a domain $D_{j} \subset L_{j}, j=1,2$. There is then $\tau>0$ such that if $0<t \leq \tau$ and if $E$ is a unitary map of $\mathbb{C}^{2}$ to itself with $|E-I|<\tau$, then $E\left(V_{t}\right)$ is transverse to $b \Omega$ and $D_{t E}=E\left(V_{t}\right) \cap \Omega$ is a transversely embedded analytic disc. Moreover, if $\delta>0$ is sufficiently small, then $\overline{D_{t E}} \backslash \delta \mathbb{B}$ is an arbitrarily small perturbation of $\left[\overline{D_{1}} \backslash \delta \mathbb{B}\right] \cup\left[\overline{D_{2}} \backslash \delta \mathbb{B}\right]$ provided that $t>0$ and $|E-I|$ are sufficiently small.

\section{Proof of Lemma 3.3.}

Step 1. If $r>0$ is sufficiently small, then $b(r \mathbb{B})$ is transverse to $\Lambda_{1}$ and to $\Lambda_{2}$, and each $\Lambda_{i}$ intersects $b(r \mathbb{B})$ at exactly two points. In addition, $b(r \mathbb{B})$ intersects $b \Omega$ transversely in a slight perturbation of $H \cap b(r \mathbb{B})$ where $H=\{R e(z+w)=0\}$. Both $b(r \mathbb{B})$ and $b \Omega$ are transverse to $L_{1}$ and $L_{2}$. For each $i, D_{i} \backslash r \overline{\mathbb{B}}$ is bounded by the union of an $\operatorname{arc} \lambda_{i}=\Lambda_{i} \backslash r \mathbb{B}$ and a circular arc $\gamma_{i}$ contained in $b(r \mathbb{B})$.

On any compact set missing the origin $E\left(V_{t}\right)$ is an arbitrarily small perturbation of $L_{1} \cup L_{2}$ provided that $t>0$ and $|E-I|$ are small enough. In this case, $b \Omega$ and $b(r \mathbb{B})$ cut out of $V_{t}$ two domains $D_{i}(t, E, r)$, and by the transversality mentioned above, each $D_{i}(t, E, r)$ is a slight perturbation of $D_{i} \backslash r \overline{\mathbb{B}}$. It is bounded by two smooth arcs, one lying near $\lambda_{i}$, the other near $\gamma_{i}$.

Suppose for the moment that we have proved that

(B) there is $\tau>0$ such that for each $t, 0<t \leq \tau, E\left(V_{t}\right)$ is transverse to $b \Omega$ and $E\left(V_{t}\right) \cap b \Omega$ is a simple closed curve $\Lambda_{E t}$.

For $0<t \leq \tau$ define $D_{t E}$ to be $\Omega \cap E\left(V_{t}\right)$. By Lemma 3.2 each $D_{t E}$ is a transversely embedded analytic disc.

Step 2. We first describe what we are going to do to prove (B). If $V_{0}=\{z w=0\}=L_{1} \cup L_{2}$, then $V_{0} \cap b \Omega$ is a figure eight, a union of two simple closed curves, $\Lambda_{1}$ and $\Lambda_{2}$, in $\mathbb{C}^{2}$ which meet only at the origin. (Note that they are not tangent to each other there.) Fix a small ball $\mathcal{B}$ centered at the origin. Outside $\mathcal{B}$ the variety $V_{t}$ is a small perturbation of $V_{0}$ when $t>0$ is small enough. It follows that $b \Omega \cap E\left(V_{t}\right) \backslash \mathcal{B}$ is, for small $t>0$, a small perturbation of $\left(\Lambda_{1} \backslash \mathcal{B}\right) \cup\left(\Lambda_{2} \backslash \mathcal{B}\right)$, which is a union to two disjoint arcs. Now we use Lemma 2.5 to analyze what happens in $\mathcal{B}$ as we pass from 
$t=0$ to a small positive $t$. A careful analysis will show that $\mathcal{B} \cap V_{0}$, the cross in the figure eight, gets replaced with two arcs that connect the endpoints of the two arcs whose union is $\left[b \Omega \cap E\left(V_{t}\right)\right] \backslash \mathcal{B}$ in such a way that the union of all four arcs is a simple closed curve.

Now let us give the details of the proof of (B). Let $\delta>0$ be smaller than $\tau$ in Lemma 2.2. Since $\operatorname{Re}(z+w)=0$ is tangent to $b \Omega$ at 0 , it follows that there are $r>0$ and a smooth function $\psi$ on $\mathbb{R}^{3}$ such that $\psi(0)=0,|(D \psi)(p)|<$ $\frac{\delta}{2}\left(p \in \mathbb{R}^{3}\right)$ and such that $\mathbb{B}(0, r) \cap G r(\psi)=\mathbb{B}(0, r) \cap b \Omega$. There is a $\nu>0$ such that if $E$ is a unitary map with $|E-I|<\nu$, then $E(b \Omega)$ is transverse to both $L_{1}$ and $L_{2}$, and there is a unique function $\psi_{E}$, smooth on $\mathbb{R}^{3}$ with $\psi_{E}(0)=0,\left|\left(D \psi_{E}\right)(p)\right|<\delta\left(p \in \mathbb{R}^{3}\right)$ and such that $E(G r(\psi))=G r\left(\psi_{E}\right)$.

Provided that $\delta$ has been chosen small enough Lemma 2.2 shows that $G r\left(\psi_{E}\right) \cap L_{i}=\left\{\left(\Phi_{i E}(V), V\right): V \in \mathbb{R}\right\}$ where $\Phi_{i E}=\left(X_{i E}, Y_{i E}, U_{i E}\right)$ is smooth on $\mathbb{R}$ and $\Phi_{1 E}^{\prime}(V)$ is arbitrarily close to $(0,-1,0)$, and $\Phi_{2 E}^{\prime}(V)$ is arbitrarily close to $(0,1,0)$ uniformly in $V \in \mathbb{R}$ provided that $\delta$ and $\gamma$ are small enough. In particular, we may assume that for $V \in \mathbb{R}$

$$
\left|X_{i E}^{\prime}(V)\right|<\frac{1}{2},\left|U_{i E}^{\prime}(V)\right|<\frac{1}{2},-\frac{3}{2}<Y_{1 E}^{\prime}(V)<-\frac{1}{2}, \frac{1}{2}<Y_{2 E}^{\prime}(V)<\frac{3}{2} .
$$

Thus, $Y_{1 E}$ is strictly decreasing, $Y_{2 E}$ is strictly increasing, and since $Y_{i E}(0)=$ 0 it follows that $Y_{1 E}(V)>0$ and $Y_{2 E}(V)<0$ when $V<0$ while $Y_{1 E}(V)<0$ and $Y_{2 E}(V)>0$ when $V>0$.

Step 3. We have to take into account that $\operatorname{Gr}\left(\psi_{E}\right)$ coincides with $E(\Omega)$ only within $\mathbb{B}(0, r)$. With $M$ as in Lemma 2.5 , choose $\omega>0$ so small that if

$$
\mathcal{A}=\{|X|,|Y|,|U|<(M+2) \omega \text { and }|V|<\omega\}
$$

then $\mathcal{A} \subset B(0, R)$. The bounds (3.3) imply that

$$
\left|X_{i E}( \pm \omega)\right|<\frac{1}{2} \omega,\left|U_{i E}( \pm \omega)\right|<\frac{1}{2} \omega \text {, and }\left|Y_{i E}( \pm \omega)\right|<\frac{3}{2} \omega .
$$

As $\delta$ is smaller than the $\tau$ of Lemma 2.5, this lemma implies that for each $t>0$,

$$
G r\left(\psi_{E}\right) \cap V_{t}=\left\{\left(\Phi_{1 E t}(V), V\right): V \in \mathbb{R}\right\} \cup\left\{\left(\Phi_{2 E t}(V), V\right): V \in \mathbb{R}\right\}
$$

where $\Phi_{i E t}=\left(X_{i E t}, Y_{i E t}, U_{i E t}\right)$ is smooth on $\mathbb{R}$ and $Y_{1 E t}(V)>0$, and $Y_{2 E t}(V)<0$ for all $V \in \mathbb{R}$.

If $t_{0}>0$ and $\gamma>0$ are sufficiently small, then by transversality, the set $\left\{\Phi_{1 E t}(\omega), \Phi_{2 E t}(\omega)\right\}$ is a small perturbation of the set $\left\{\Phi_{1 I}(\omega), \Phi_{2 I}(\omega)\right\}$ and the set $\left\{\Phi_{1 E t}(-\omega), \Phi_{2 E t}(-\omega)\right\}$ is a small perturbation of the set $\left\{\Phi_{1 I}(-\omega)\right.$, $\left.\Phi_{2 I}(-\omega)\right\}$ whenever $0<t \leq t_{0}$ and $|E-I|<\gamma$. In particular by (3.4) we may suppose that

$$
\left|X_{i E t}( \pm \omega)\right|<\omega,\left|U_{i E t}( \pm \omega)\right|<\omega \text { and }\left|Y_{i E t}( \pm \omega)\right|<2 \omega .
$$


Since by Lemma $2.5\left|X_{i E t}^{\prime}\right|,\left|Y_{i E t}^{\prime}\right|,\left|U_{i E t}^{\prime}\right|<M$ on $\mathbb{R}$, (3.5) implies that for $|V|<\omega$,

$$
\left|X_{i E t}(V)\right|<(M+1) \omega,\left|U_{i E t}(V)\right|<(M+1) \omega, \text { and }\left|Y_{i E t}(V)\right|<(M+2) \omega
$$

so, provided that $t_{0}$ and $\gamma$ are small enough $\left(X_{i E t}(V), Y_{i E t}, U_{i E t}(V), V\right) \in \mathcal{A}$ when $|V|<\omega$. Since $\mathcal{A} \subset \mathbb{B}(0, r)$ where $G r\left(\psi_{E}\right)$ coincides with $E(b \Omega)$, it follows that $\mathbb{B}(0, r) \cap G r\left(\psi_{E}\right) \cap V_{t} \cap\{|V|<\omega\}=\mathbb{B}(0, r) \cap E(b \Omega) \cap V_{t} \cap\{|V|<$ $\omega\}$.

To see that $E\left(V_{t}\right) \cap b \Omega$ is a simple closed curve if $t>0$ and $|E-I|$ are small is equivalent to seeing that $V_{t} \cap E(b \Omega)$ is a simple closed curve provided that $t>0$ and $|E-I|$ are small.

To see that $V_{t} \cap E(b \Omega)$ is a simple closed curve, observe first that $\Lambda_{i}=\Lambda_{i I}$ consists of a short arc $\Lambda_{i}^{s}=\left\{\left(\Phi_{i I}(V), V\right):|V|<\omega\right\}$ and a long arc $\Lambda_{i}^{\ell}$, which joins the points $\left(\Phi_{i I}(\omega), \omega\right)$ and $\left(\Phi_{i I}(-\omega),-\omega\right)$. If $\omega>0$ is small enough then the long arc $\Lambda_{E}^{\ell}$ meets $\overline{\mathcal{A}}$ only at its endpoints at which it is transverse to the hyperplanes $V=\omega$ and $V=-\omega$, respectively. This transversality, together with the transversality of $L_{1}$ and $L_{2}$ to $b \Omega$ implies that when $t=0$ changes to $t, 0<t \leq t_{0}$ and $I$ changes to $E,|E-I|<\gamma$, then, provided that $t_{0}$ and $\gamma$ are small enough, the long arc $\Lambda_{i}^{\ell}$ will change arbitrarily little to an $\operatorname{arc} \Lambda_{i E t}^{\ell}$ with endpoints $\left(T_{i E t}^{+}, \omega\right)$ close to $\left(\Phi_{i I}(\omega), \omega\right)$ and $\left(T_{i E t}^{-},-\omega\right)$ close to $\left(\Phi_{i I}(-\omega),-\omega\right)$, which will still meet $\overline{\mathcal{A}}$ only at its endpoints. In particular,

(C) the $Y$-coordinates of $T_{1 E t}^{-}$and $T_{2 E t}^{+}$will be positive, and the $Y$ coordinates of $T_{1 E t}^{+}$and $T_{2 E t}^{-}$will be negative.

We have $\Lambda_{1 E t}^{\ell} \cup \Lambda_{2 E t}^{\ell}=\left[V_{t} \cap E(b \Omega)\right] \backslash \mathcal{A}$.

On the other hand, provided that $t_{0}>0$ and $\gamma$ are small enough, for every $E,|E-I|<\gamma$, and for every $t, 0<t \leq t_{0}, \mathcal{A} \cap E(b \Omega) \cap V_{t}=\mathcal{A} \cap$ $G r\left(\psi_{E}\right) \cap V_{t}=\left\{\left(\Phi_{1 E t}(V), V\right):|V|<\omega\right\} \cup\left\{\left(\Phi_{2 E t}(V), V\right):|V|<\omega\right\}$ where $Y_{1 E t}(V)>0$, and $Y_{2 E t}(V)<0$ for all $V \in \mathbb{R}$. Now $V_{t}$ intersects $E(b \Omega) \backslash \mathcal{A}$ transversely provided that $\gamma$ and $t_{0}$ are small enough, and by Lemma $2.5, V_{t}$ meets $E(b \Omega) \cap \mathcal{A}$ transversely. Thus $E\left(V_{t}\right)$ intersects $b \Omega$ transversely, which implies that $E\left(V_{t}\right) \cap b \Omega$ is a closed, one-dimensional submanifold of $\mathbb{C}^{2}$. As $V_{t} \cap E(b \Omega) \backslash \mathcal{A}=\Lambda_{1 E t}^{\ell} \cup \Lambda_{2 E t}^{\ell}$ and $V_{t} \cap(b \Omega) \cap \mathcal{A}=\left\{\left(\Phi_{1 E t}(V), V\right):|V|<\right.$ $\omega\} \cup\left\{\left(\Phi_{2 E t}(V), V\right):|V|<\omega\right\}$, it follows that

$$
\begin{aligned}
E(b \Omega) \cap V_{t}=\Lambda_{1 E t}^{\ell} \cup \Lambda_{2 E t}^{\ell} \cup\left\{\left(\Phi_{1 E t}(V),\right.\right. & V):|V|<\omega\} \\
& \cup\left\{\left(\Phi_{2 E t}(V), V\right):|V|<\omega\right\} .
\end{aligned}
$$

The last two arcs are contained in $\mathcal{A}$, and the first two miss $\mathcal{A}$. As $\Lambda_{1}^{\ell} \cap \Lambda_{2}^{\ell}=$ $\emptyset$, it follows that $\Lambda_{1 E t}^{\ell} \cap \Lambda_{2 E t}^{\ell}=\emptyset$ if $t_{0}$ and $\gamma$ are small enough. The last two arcs in (3.6) are also disjoint since $Y_{1 E t}(V)>0$ and $Y_{2 E t}(V)<0$ for all $V \in \mathbb{R}$. This, together with (C), implies that the only way for the right side 
of (3.6) to be a closed submanifold of $\mathbb{C}^{2}$ is for

$$
\Phi_{1 E t}(-\omega)=T_{1 E t}^{-} \quad \Phi_{1 E t}=T_{2 E t}^{+}
$$

and

$$
\Phi_{2 E t}(-\omega)=T_{2 E t}^{-} \quad \Phi_{2 E t}(\omega)=T_{1 E t}^{+} .
$$

Thus, $E(b \Omega) \cap V_{t}$ consists of the long arc $\Lambda_{1 E t}^{\ell}$ joining $\left(T_{1}^{+}, \omega\right)$ with $\left(T_{1}^{-},-\omega\right)$ followed by the arc $\left\{\left(\Phi_{1 E t}(V), V\right):|V|<\omega\right\}$ joining $\left(T_{1}^{-},-\omega\right)$ with $\left(T_{2}^{+}, \omega\right)$ followed by the long arc $\Lambda_{2 E t}^{\ell}$ joining $\left(T_{2}^{+}, \omega\right)$ with $\left(T_{2}^{-},-\omega\right)$ followed finally by the arc $\left\{\left(\Phi_{E t}(V), V\right):|V|<\omega\right\}$ joining $\left(T_{2}^{-},-\omega\right)$ with $\left(T_{1}^{+}, \omega\right)$. This proves that $E(b \Omega) \cap V_{t}$ is a simple closed curve provided that $t>0$ and $|E-I|$ are small enough.

This completes the proof of Lemma 3.3.

Discussion 1. Given $\epsilon>0$, the $\omega$ in the proof of Lemma 3.3 can be chosen so small that $4 \omega \sqrt{3 M^{2}+1}<\epsilon$. This implies that the length of $E(b \Omega) \cap \mathcal{A} \cap V_{t}$ does not exceed $\epsilon$. Indeed, length $\left(E(b \Omega) \cap \mathcal{A} \cap V_{t}\right)=\operatorname{length}\left(V_{t} \cap\right.$ $\left.G r\left(\psi_{E}\right) \cap \mathcal{A}\right)=\int_{-\omega}^{\omega}\left[\sum_{j=1}^{2}\left[X_{j E t}^{\prime}(v)^{2}+Y_{j E t}^{\prime}(v)^{2}+V_{j E t}^{\prime}(v)^{2}+1\right]\right]^{\frac{1}{2}} d v$, and since $\left|X_{j E t}^{\prime}(v)\right|,\left|Y_{j E t}^{\prime}(v)\right|,\left|V_{j E t}^{\prime}(v)\right|$ are all bounded by $M$, it follows that length $\left(E(b \Omega) \cap \mathcal{A} \cap V_{t}\right) \leq 4 \omega \sqrt{3 M^{2}+1}<\epsilon$.

Discussion 2. The implicit mapping theorem implies that given an interior point $w$ of the long arc $\Lambda_{i}^{\ell}$, there are a neighborhood $W(w)$ of $w$, a $\nu(w)>0$, and a $t_{0}(w)>0$ such that $|E-I|<\nu(w)$ and $0<t \leq t_{0}(w)$ imply that $\Lambda_{i E t}^{\ell} \cap W(w)$ is a smooth graph over $\Lambda_{i}^{\ell} \cap W(w)$. These graphs depend smoothly on $E$ and $t$. The same is true at the endpoints of $\Lambda_{i}^{\ell}$. Since we want the endpoints of $\Lambda_{i E t}^{\ell}$ to belong to $|V|=\omega$, we have to write, e.g., in a sufficiently small neighborhood $W(w)$ of $w=\left(\Phi_{i I}(\omega), \omega\right), \quad \Lambda_{i I} \cap W(w)=$ $\left\{\left(\Phi_{i I}(t), t\right): \omega \leq t<\omega+\gamma\right\}$ for some small $\gamma>0$, and then $\Lambda_{i E t}^{\ell} \cap W(w)=$ $\left\{\left(\Phi_{i E t}(t), t\right): \omega \leq t<\omega+\gamma\right\}$. A partition of unity argument together with the compactness of $\Lambda_{i}^{\ell}$ shows that given a smooth 1 -form $\alpha$ on $\mathbb{C}^{2}$, we have

$$
\lim _{E \rightarrow I, t \rightarrow 0} \int_{\Lambda_{i E t}^{\ell}} \alpha=\int_{\Lambda_{i}^{\ell}} \alpha .
$$

Similar reasoning applies to show that if $t_{0}$ and $\nu$ are small enough, then the lengths of $\Lambda_{i E t}^{\ell},|E-I|<\nu, 0<t \leq t_{0}$, are uniformly bounded. We already know that the lengths of $E(b \Omega) \cap V_{t} \cap \mathcal{A}$ are uniformly bounded. Thus, the lengths of $E(b \Omega) \cap V_{t}$ are uniformly bounded provided that $|E-I|<\nu, 0<$ $t \leq t_{0}$.

Discussion 3. Let $\pi_{1}(z, w)=z, \pi_{2}(z, w)=w$ be the coordinate projections in $\mathbb{C}^{2}$. For each $t, 0<t<\tau$, let $D_{t E}^{i}=\pi_{i}\left(E^{-1}\left(D_{t E}\right)\right), i=1$, 2. Since the $E^{-1}\left(D_{t E}\right)$ are discs in $V_{t}, 0<t<\tau$, the $D_{t E}^{i}$ are Jordan domains in $\mathbb{C}$, 
bounded by simple closed curves $\pi_{i}\left(E\left(V_{t}\right) \cap b \Omega\right)$, which, when $t \rightarrow 0, E \rightarrow$ $I$, by the last statement of Lemma 3.3, converge to $D_{i}$ in the following sense: Given $\varepsilon>0$ there is a $\delta, 0<\delta<\tau$, such that if $0<t<\delta$ and $|E-I|<\delta$ then there is a homeomorphism $\psi_{t E}^{i}: b D_{i} \rightarrow b D_{t E}^{i}$ such that $\left|\psi_{t E}^{i}(w)-w\right|<\varepsilon\left(w \in b D_{i}\right), i=1,2$. This implies [Po, p. 26] that given a conformal map $\varphi_{1}: \Delta \rightarrow D_{1}$ and $\varepsilon>0$ there is a $\delta, 0<\delta<\tau$, such that whenever $0<t<\delta$ and $|E-I|<\delta$, there is a conformal map $\varphi_{t E}^{1}: \Delta \rightarrow$ $D_{t E}^{1}$ such that $\left|\varphi_{t E}^{1}-\varphi_{1}\right|<\varepsilon$ on $\Delta$ which further implies that there is a parametrization $\zeta \mapsto\left(\varphi_{t E}^{1}(\zeta), t^{2} / \varphi_{t E}^{1}(\zeta)\right)=\Phi_{t E}(\zeta)$ of $E^{-1}\left(D_{t E}\right)$ such that $\left|\Phi_{t E}(\zeta)-\Phi_{1}(\zeta)\right|<2 \varepsilon+\operatorname{diam}\left(D_{2}\right)(\zeta \in \Delta)$ whenever $0<t<\delta,|E-I|<\delta$ where $\Phi_{1}(\zeta)=\left(\varphi_{1}(\zeta), 0\right)$ is a parametrization of $D_{1}$. In particular, given a neighbourhood $\mathcal{D}$ of $D_{1}$ in the space of transversely embedded analytic discs, $D_{t E} \in \mathcal{D}(0<t<\delta,|E-I|<\delta)$ provided that $\operatorname{diam}\left(D_{2}\right)$ and $\delta$ are small enough.

Let $\alpha$ be a continuous 1 -form on $C^{2}$ with compact support. Given $\epsilon>0$, there is a $\delta>0$ such that for every smooth compact curve $\Sigma$ in $\mathbb{C}^{2}$, we have

$$
\left|\int_{E(\Sigma)} \alpha-\int_{\Sigma} \alpha\right|=\left|\int_{\Sigma} E^{*} \alpha-\alpha\right|<\epsilon \cdot \operatorname{length}(\Sigma)
$$

whenever $E$ is a unitary map with $|E-I|<\delta$.

Lemma 3.4. Let $\Omega, \Lambda_{1}, \Lambda_{2}$ and $V_{t}, t>0$, be as in Lemma 3.3. If $\alpha$ is a continuous 1 -form on $\mathbb{C}^{2}$, then

$$
\lim _{E \rightarrow I, t \rightarrow 0} \int_{b \Omega \cap E\left(V_{t}\right)} \alpha=\int_{\Lambda_{1} \cup \Lambda_{2}} \alpha=\int_{b \Omega \cap V_{0}} \alpha .
$$

Proof. With no loss of generality we suppose that $\alpha$ has compact support. Suppose that we have proved that

$$
\lim _{E \rightarrow I, t \rightarrow 0} \int_{E(b \Omega) \cap V_{t}} \alpha=\int_{\Lambda_{1} \cup \Lambda_{2}} \alpha .
$$

Since the lengths of $E(b \Omega) \cap V_{t},|E-I|<\delta, 0<t<t_{0}$, are uniformly bounded, the preceding discussion implies that given $\epsilon>0$ there is a $\delta>0$ such that

$$
\left|\int_{\tilde{E}\left(E(b \Omega) \cap V_{t}\right)} \alpha-\int_{E(b \Omega) \cap V_{t}} \alpha\right|<\epsilon
$$

whenever $|\tilde{E}-I|<\delta,|E-I|<\gamma$, and $0<t<t_{0}$. In particular, taking $\tilde{E}=E^{-1}$ yields

$$
\left|\int_{b \Omega \cap E^{-1}\left(V_{t}\right)} \alpha-\int_{E(b \Omega) \cap V_{t}} \alpha\right|<\epsilon
$$


whenever $\left|E^{-1}-I\right|<\delta,|E-I|<\gamma, 0<t<t_{0}$. Now $E \rightarrow I$ is equivalent to $E^{-1} \rightarrow I$, so

$$
\lim _{E^{-1} \rightarrow I, t \rightarrow 0} \int_{b \Omega \cap E^{-1}\left(V_{t}\right)} \alpha=\lim _{E \rightarrow I, t \rightarrow 0} \int_{E(b \Omega) \cap V_{t}} \alpha
$$

provided that the limit on the right exists. Thus (3.9) implies (3.8). It remains to prove (3.9).

Let $\epsilon>0$, and let $L$ be a uniform bound for the coefficients of $\alpha$. By the Discussion 1 above, one can choose $\omega>0, t_{o}>0$, and $\nu>0$ such that

$$
\text { length }\left(E(b \Omega) \cap \mathcal{A} \cap\left(\Lambda_{1} \cup \Lambda_{2}\right)\right)<\frac{\epsilon}{16 L}
$$

and

$$
\operatorname{length}\left(E(b \Omega) \cap \mathcal{A} \cap V_{t}\right)<\frac{\epsilon}{16 L}
$$

whenever $|E-I|<\nu$ and $0<t<t_{0}$. It follows that when $0<t<t_{0}$ and $|E-I|<\nu$,

$$
\left|\int_{E(b \Omega) \cap \mathcal{A} \cap V_{t}} \alpha\right|<\frac{\epsilon}{4} \text { and }\left|\int_{E(b \Omega) \cap \mathcal{A} \cap\left(\Lambda_{1} \cup \Lambda_{2}\right)} \alpha\right|<\frac{\epsilon}{4} .
$$

Further, by (3.7), we can pass to smaller $\nu>0$ and $t_{0}>0$ if necessarily to get

$$
\left|\int_{\Lambda_{i E t}^{\ell}} \alpha-\int_{\Lambda_{i}^{\ell}} \alpha\right|<\frac{\epsilon}{4} \quad\left(0<t<t_{o},|E-I|<\nu, i=1,2\right) .
$$

Thus, if $0<t<t_{0}$ and $|E-I|<\nu$, then (3.10) and (3.11) imply that

$$
\begin{aligned}
\left|\int_{E(b \Omega) \cap V_{t}} \alpha-\int_{\Lambda_{1} \cup \Lambda_{2}} \alpha\right| \leq & \sum_{i=1}^{2}\left|\int_{\Lambda_{i E t}^{\ell}} \alpha-\int_{\Lambda_{i}^{\ell}} \alpha\right|+\left|\int_{E(b \Omega) \cap \mathcal{A} \cap V_{t}} \alpha\right| \\
& +\left|\int_{E(b \Omega) \cap \mathcal{A} \cap\left(\Lambda_{1} \cup \Lambda_{2}\right)} \alpha\right| \leq \epsilon .
\end{aligned}
$$

This proves (3.9). The proof of Lemma 3.4 is complete.

Lemma 3.5. Let $\Omega \subset \mathbb{C}^{2}$ be a bounded domain with $0 \in b \Omega$. Assume that $b \Omega$ is of class $\mathcal{C}^{2}$ in a neighborhood $U$ of $\left(L_{1} \cup L_{2}\right) \cap b \Omega, L_{1}, L_{2}$ the coordinate axes, and that $L_{1}, L_{2}$ intersect $b \Omega$ transversely so that $D_{j}=L_{j} \cap \Omega$ are transversely embedded analytic discs, $j=1,2$. Let $w_{1} \in D_{1}$. There is then a sequence $\left\{A_{n}\right\}_{n=1,2, \ldots} \subset \Omega$ of transversely embedded analytic discs such that $w_{1} \in A_{n}, b A_{n} \subset U(n \in \mathbb{N})$ and such that for each continuous 1-form $\alpha$ on $\mathbb{C}^{2}$

$$
\lim _{n \rightarrow \infty} \int_{b A_{n}} \alpha=\int_{b D_{1}} \alpha+\int_{b D_{2}} \alpha
$$


Proof. Suppose to begin with that $b \Omega$ is of class $\mathcal{C}^{2}$.

Assume first that $\operatorname{Re}(z+w)=0$ is the tangent space to $b \Omega$ at 0 . By Lemma 3.3 there is $\tau>0$ such that $b \Omega \cap E\left(V_{t}\right)$ is a simple closed curve contained in $U$ that bounds the analytic disc $E\left(V_{t}\right) \cap \Omega$ transverse to $b \Omega$ provided that $|E-I|$ and $t$ are small enough. Since on a compact set missing $0, V_{t}$ is an arbitrarily small perturbation of $L_{1} \cup L_{2}$ provided that $t$ is small enough, it follows that given $\tau_{n}\left(\tau_{n}<\tau\right)$, there are $t_{n}, 0<t_{n}<\tau_{n}$ and $E_{n},\left|E_{n}-I\right|<\tau_{n}$, such that $w_{1} \in E_{n}\left(V_{t_{n}}\right)$. Put $A_{n}=E_{n}\left(V_{t_{n}}\right) \cap \Omega$. Then the $A_{n}$ are transversely embedded analytic discs and by Lemma 3.4, (3.12) holds for every continuous 1 -form on $\mathbb{C}^{2}$.

In the general case, let $\operatorname{Re}(p z+q w)=0$ be the equation of the tangent space to $b \Omega$ at 0 . Since $L_{1}, L_{2}$ are transverse to $b \Omega$ it follows that $p \neq 0, q \neq$ 0 , so $F(z, w)=\left(\frac{z}{p}, \frac{w}{q}\right)$ is an isomorphism of $\mathbb{C}^{2}$ with $F(0)=0, F\left(L_{i}\right)=$ $L_{i}, i=1,2$, and $\operatorname{Re}(z+w)=0$ is the tangent space to $b \tilde{\Omega}$ if $\tilde{\Omega}=F(\Omega)$. We are now in the situation above with $\Omega$ replaced by $\tilde{\Omega}$ and with $D_{j}$ replaced by $\tilde{D}_{j}=L_{j} \cap \tilde{\Omega}$. Thus, there is a sequence $\tilde{A}_{n} \subset \tilde{\Omega}$ of transversely embedded analytic discs such that $b \tilde{A}_{n} \subset F(U), F\left(w_{1}\right) \in \tilde{A}_{n}$ and such that

$$
\lim _{n \rightarrow \infty} \int_{b \tilde{A}_{n}} \beta=\int_{b \tilde{D}_{1}} \beta+\int_{b \tilde{D}_{2}} \beta
$$

for every continuous 1 -form $\beta$ on $\mathbb{C}^{2}$. In particular given a continuous 1 -form $\alpha$ on $\mathbb{C}^{2}$ it follows that if $\beta=\left(F^{-1 *}\right) \alpha$ then

$$
\lim _{n \rightarrow \infty} \int_{b A_{n}} F^{*}\left(F^{-1 *}\right) \alpha=\int_{b D_{1}} F^{*}\left(F^{-1 *}\right) \alpha+\int_{b D_{2}} F^{*}\left(F^{-1 *}\right) \alpha
$$

where $A_{n}=F^{-1}\left(\tilde{A}_{n}\right)$, which implies (3.12).

If the boundary $b \Omega$ is of class $\mathcal{C}^{2}$ only in a neighborhood of the intersection $\left(L_{1} \cup L_{2}\right) \cap b \Omega$, then a small modification of the argument just given is required. The set $U$ can be taken to lie in the subset of $b \Omega$ that is a manifold of class $\mathcal{C}^{2}$. Then, in the proof of Lemma 3.3 it is enough to assume that $b \Omega$ is of class $\mathcal{C}^{2}$ only in a neighborhood of $\left(L_{1} \cup L_{2}\right) \cap b \Omega$, since we are there intersecting $b \Omega$ with varieties that are small perturbations of $L_{1} \cup L_{2}$.

Lemma 3.5 is proved.

Discussion. Again, as in Discussion 3 after Lemma 3.3, given a neighbourhood $\mathcal{D}$ of $D_{1}$ in the space of transversely embedded analytic discs, $A_{n}$ can be chosen to belong to $\mathcal{D}$ provided that diam $\left(D_{2}\right)$ is small enough.

We now continue the proof of Theorem 1.1, but no longer under the restriction to domains in $\mathbb{C}^{2}$. We are dealing with the case that $\Omega$ is strictly convex at $z_{0}$.

Lemma 3.6. Let $\Omega$ be a bounded domain in $\mathbb{C}^{N}$ with $b \Omega$ of class $\mathcal{C}^{2}$. Suppose that $D_{0} \subset \Omega$ is a transversely embedded analytic disc. Let $w_{0} \in D_{0}$ and $z_{0} \in$ 
$b D_{0}$, and suppose that $\Omega$ is strictly convex at $z_{0}$. Let $\mathcal{D}$ be a neighbourhood of $D_{0}$ in the space of transversely embedded analytic discs. Denote by $\Lambda\left(z_{0}\right)$ a complex line that is tangent to $b \Omega$ at $z_{0}$. There are an open ball $B$ centered at $z_{0}$ and a neighborhood $\mathcal{L}$ of $\Lambda\left(z_{0}\right)$ in the space of all complex lines in $\mathbb{C}^{N}$ such that for each $L \in \mathcal{L}$ that meets $\Omega \cap B$

(a) $L \cap B \cap b \Omega$ is a compact convex curve, and

(b) there are $A \in \mathcal{D}$ and a sequence $\left\{A_{n}\right\}_{n=1,2, \ldots} \subset \mathcal{D}$ such that $w_{0} \in$ $A, w_{0} \in A_{n}(n \in \mathbb{N})$ and such that for each smooth 1-form $\alpha$ on $\mathbb{C}^{2}$

$$
\lim _{n \rightarrow \infty} \int_{b A_{n}} \alpha=\int_{b A} \alpha+\int_{L \cap Q \cap b \Omega} \alpha .
$$

As in the two-dimensional analysis, this implies that $\int_{L \cap Q \cap b \Omega} f \omega=0$. Granted this lemma, Theorem 1.1, in the case of convexity, now follows from the following lemma.

Lemma 3.7. Let $D$ be a bounded convex domain with $b D$ of class $\mathcal{C}^{2}$ and strictly convex at $z_{0} \in b D$. Let $\mathcal{L}$ be an open set of complex lines in $\mathbb{C}^{N}$ that contains a line tangent to $b D$ at $z_{0}$. If $f$ is a continuous function on $b D$ that with the property that $\int_{L \cap b D} f \alpha=0$ whenever $L \in \mathcal{L}$ meets $D$ and whenever $\alpha \in \mathbb{C}^{1 ; 0}[d z]$, then $f$ is a CR-function on a neighborhood of $z_{0}$ in $b D$.

Proof. As $\mathcal{L}$ contains $L_{0}$, a complex line tangent to $b D$ at $z_{0}$ and is open, the result follows, in the case that $N=2$, from a result in [G12]. In the case of arbitrary $N$, a different analysis is necessary.

Thus, consider the case of general $N$. Let $H_{0}$ be the real hyperplane tangent to $b D$ at $z_{0}$, and let $T_{0} \subset H_{0}$ be the complex hyperplane in $\mathbb{C}^{N}$ that goes through $z_{0}$. We shall show that if $T$ is a complex hyperplane in $\mathbb{C}^{N}$ that is near $T_{0}$ and that meets $D$, then $\int_{T \cap b D} f \vartheta=0$ for all $(N, N-2)$-forms $\vartheta$ on $\mathbb{C}^{N}$ with constant coefficients. Granted this, the result we want is a consequence of Theorem 3.2.1 of [GIS].

The complex hyperplane $T_{0}$ is a disjoint union of complex lines parallel to $L_{0}$. Continuity and the openness of $\mathcal{L}$ imply the existence of an open set $\mathcal{T}$ in the space of complex hyperplanes in $\mathbb{C}^{N}$ such that $T_{0} \in \mathcal{T}$ and such that each $T \in \mathcal{T}$ is a union of complex lines $L$ each of which is parallel to an element of $\mathcal{L}$ and each of which is either disjoint from $\bar{D}$, meets $\bar{D}$ in a single point, or else meets $b D$ in a small convex curve lying near $z_{0}$. If $\mathcal{T}$ is small enough, then as $\mathcal{L}$ is open, each $T \in \mathcal{T}$ is a union of lines $\left\{L_{\lambda}\right\}_{\lambda \in \Lambda(T)}$ parallel to an element of $\mathcal{L}$ with the additional property that if $L_{\lambda}$ meets $b D$, then $L_{\lambda} \in \mathcal{L}$.

By hypothesis, for a given $T \in \mathcal{T}, \int_{L_{\lambda} \cap b D} f \omega=0$ for every $\lambda \in \Lambda(T)$. Lemma 2.2.1 of [GlS] implies that for each $T \in \mathcal{T}, \int_{T \cap b D} f \vartheta=0$ for each $\vartheta$, an $(N, N-2)$-form on $\mathbb{C}^{N}$ with constant coefficients. The lemma is proved. 
The proof of Lemma 3.6 depends on the following simple observation.

Lemma 3.8. Let $\delta>0$, and let $V$ be a closed one-dimensional complex submanifold of an open set in $\mathbb{C}^{N}$ such that for each $\zeta,|\zeta|<\delta, V$ intersects the hyperplane $H_{\zeta}=\left\{z: z_{1}=\zeta\right\}$ at one point and transversely. Assume that $V$ meets $H_{0}$ at the origin. There is a biholomorphic map $\Phi$ of $\left\{z \in \mathbb{C}^{N}\right.$ : $\left.\left|z_{1}\right|<\delta\right\}$ onto itself that fixes $H_{0}$ and has the property that $\Phi\left(V \cap\left\{\left|z_{1}\right|<\right.\right.$ $\delta\})=\{(\zeta, 0, \ldots, 0):|\zeta|<\delta\}$.

Proof. Let $V \cap H_{\zeta}=\varphi(\zeta)=\left(\zeta, \varphi_{2}(\zeta), \ldots, \varphi_{N}(\zeta)\right), \quad|\zeta|<\delta$. The map $\varphi: \delta \Delta \rightarrow \mathbb{C}^{N}$ is holomorphic by the transversality. As it satisfies $\varphi(0)=0$ the map $\Phi$ given by

$$
\Phi\left(z_{1}, \ldots, z_{N}\right)=\left(z_{1}, z_{2}-\varphi_{2}\left(z_{1}\right), \ldots, z_{N}-\varphi_{N}\left(z_{1}\right)\right)
$$

has the desired properties.

Proof of Lemma 3.6. The idea is very simple; we describe it in a special case. Since $\Omega$ is strictly convex at $z_{0}$ there are an open ball $B$ centered at $z_{0}$ and a neighbourhood $\mathcal{L}$ of $\Lambda\left(z_{0}\right)$ in the space of complex lines in $\mathbb{C}^{N}$ such that if $L \in \mathcal{L}$ and $L \cap B$ meets $\Omega$ then $L \cap B$ meets $b \Omega$ transversely in a compact convex curve. Moreover, given a neighbourhood $\mathcal{E}$ of $z_{0}$ in $\mathbb{C}^{N}$, $L \cap B \cap \bar{\Omega} \subset \mathcal{E}$ provided that $\mathcal{L}$ is small enough. Let $V$ be a one dimensional submanifold of an open neighbourhood of $\bar{\Omega}$ such that $V \cap \Omega=D_{0}$. We find a biholomorphic map $G$ from a neighbourhood $Q$ of $\overline{D_{0}}$ in $\mathbb{C}^{N}$ to a domain $G(Q)$ in such a way that $G\left(z_{0}\right)=0$ and that $G$ maps $Q \cap V$ into the $z_{1}$-axis. Shrink $\mathcal{L}$ if necessary so that $L \cap B \cap \bar{\Omega} \subset Q$ whenever $L \in \mathcal{L}$ and assume that $L \in \mathcal{L}$ and that $z_{0} \in L$. We modify $G$ so that, in addition, it maps $L \cap B \cap Q$ into the $z_{2}$-axis. Then we intersect $G(Q)$ with the two-dimensional subspace $M$ spanned by $z_{1}$ - and $z_{2}$-axes. This gives a domain in the copy $M$ of $\mathbb{C}^{2}$ to which Lemma 3.5 applies to yield a sequence $\left\{\tilde{A}_{n}\right\}$ of transversely embedded analytic discs whose boundaries $b \tilde{A}_{n}$, in the sense of that lemma, converge to $G\left(b D_{0}\right) \cup G(L \cap B \cap \Omega)$. Then $A_{n}=G^{-1}\left(\tilde{A}_{n}\right)$, and $A_{0}=D_{0}$ will do the job. Of course, we must be more careful when $L$ does not pass through $z_{0}$.

Step 1. Let $V$ be a closed one-dimensional submanifold of an open neighborhood of $\bar{\Omega}$ that intersects $b \Omega$ transversely so that $D_{0}=V \cap \Omega, b D_{0}=V \cap$ $b \Omega$. By Lemma 3.1 above, $\overline{D_{0}}$ is polynomially convex, so it has a Stein neighborhood basis. Thus, there are arbitrarily small neighborhoods $Q$ of $\bar{D}_{0}$ in $\mathbb{C}^{N}$ that are biholomorphically equivalent, say under $F$, to a domain $P$ in $\mathbb{C}^{N}$ and in such a way that $F(V \cap Q)=\left\{\left(z_{1}, \ldots, z_{N}\right) \in P: z_{2}=\cdots=z_{N}=0\right\}$, i.e., that $F(V \cap Q)$ is the intersection of $P$ with the $z_{1}$-axis. This follows from a result of Docquier and Grauert on the existence of holomorphic tubular neighborhoods -see [GR, pp. 256-257]- and the holomorphic triviality of holomorphic vector bundles over discs. The strict convexity of $\Omega$ at $z_{0}$ 
implies that there are an open ball $B$ centered at $z_{0}$ and a neighbourhood $\mathcal{L}$ of $\Lambda\left(z_{0}\right)$ in the space of complex lines such that

(D) if $L \in \mathcal{L}$ and $L \cap B$ meets $\Omega$ then $L \cap B$ meets $b \Omega$ transversely in a small compact convex curve. Moreover, given a neighbourhood $\mathcal{E}$ of $z_{0}, \mathcal{L}$ can be chosen so small that $B \cap L \cap \bar{\Omega} \subset \mathcal{E}$ for every $L \in \mathcal{L}$.

Passing to smaller $P, Q$ if necessary we may assume that there are a neighbourhood $\mathcal{T}$ of the $z_{1}$-axis in the space of all complex lines passing through $\tilde{w}_{0}=F\left(w_{0}\right)$ and a $\delta>0$ such that whenever a complex line $T^{\prime}$ is parallel to a line $T \in \mathcal{T}, \operatorname{dist}\left(T, T^{\prime}\right)<\delta$ then $T^{\prime}$ intersects $F(Q \cap b \Omega)$ transversely in a simple closed curve that bounds the domain $\mathcal{D}\left(T^{\prime}\right)=T^{\prime} \cap$ $F(Q \cap \Omega)$.

Step 2. Since $V$ is transverse to $b \Omega$ at $z_{0}$ it follows that $\Lambda\left(z_{0}\right)$ is not tangent to $V$ at $z_{0}$ so the complex tangent line to $F\left(\Lambda\left(z_{0}\right) \cap B \cap Q\right)$ at $\tilde{z}_{0}=F\left(z_{0}\right)$ does not coincide with the $z_{1}$-axis. Thus, after composing $F$ with a unitary map that fixes the $z_{1}$-axis we may, after passing to a smaller $\mathcal{L}$ and $\delta$, assume that there are a small open ball $\mathcal{E} \subset B \cap Q$ such that (D) holds, and a neighbourhood $\mathcal{H}$ of $\left\{z_{2}=0\right\}$ in the space of complex hyperplanes passing through $\tilde{w}_{0}$ such that

(E) for each $L \in \mathcal{L}, F(\mathcal{E} \cap L)$ intersects each $H^{\prime}$, a complex hyperplane parallel to an $H \in \mathcal{H}$, $\operatorname{dist}\left(H, H^{\prime}\right)<2 \delta$, at precisely one point and transversely.

By passing to a smaller $\mathcal{T}$ we can suppose that for each $T \in \mathcal{T}$ there is an $H \in \mathcal{H}$ such that $T \subset H$.

For each $T \in \mathcal{T}$ let

$$
\mathcal{D}(T, \delta)=\cup\left\{\mathcal{D}\left(T^{\prime}\right): T^{\prime} \text { parallel to } T, \operatorname{dist}\left(T, T^{\prime}\right)<\delta\right\}
$$

and

$$
P(T, \delta)=\cup\left\{T^{\prime} \cap P: T^{\prime} \text { parallel to } T, \operatorname{dist}\left(T, T^{\prime}\right)<\delta\right\} .
$$

Note that $\mathcal{D}(T, \delta)=P(T, \delta) \cap F(\Omega \cap Q)$ is a connected component of $P(T, \delta) \backslash$ $S$ where $S=F(b \Omega \cap Q)$. Choose a neighbourhood $\mathcal{P}$ of $\tilde{z}_{0}$ in $Q$ so small that

(F) if $z \in \mathcal{P}$ and if $T$ is the complex line passing through $z$ and $\tilde{w}_{0}$ then $T \in \mathcal{T}$ and $\mathcal{P} \subset \subset P(T, \delta)$.

By (D) we can pass to a smaller $\mathcal{L}$ such that

$$
L \cap B \cap \bar{\Omega} \subset Q, \quad F(L \cap B \cap \bar{\Omega}) \subset \mathcal{P} \quad(L \in \mathcal{L}) .
$$

Fix $L \in \mathcal{L}$ that meets $\Omega \cap B$. By (D) and by (3.14), $L$ meets $B \cap b \Omega$ transversely in a compact convex curve that bounds the convex domain $L \cap B \cap \Omega \subset \subset Q$. Moreover, by (3.14) we have 
(G) $F(L \cap B \cap \Omega)=F(L \cap B) \cap F(\Omega \cap Q) \subset \mathcal{P}$ is a domain in $F(L \cap B \cap Q)$ bounded by the simple closed curve $F(L \cap B \cap b \Omega)=F(L \cap B \cap Q) \cap F(b \Omega \cap$ $Q) \subset \mathcal{P}$.

Recall that

$$
P(T, \delta) \cap b \mathcal{D}(T, \delta)=P(T, \delta) \cap F(b \Omega \cap Q)
$$

and

$$
P(T, \delta) \cap F(Q \cap \Omega)=\mathcal{D}(T, \delta) .
$$

By (G) and by the fact that $\mathcal{P} \subset \subset P(T, \delta)$ it follows that $F(L \cap B \cap Q) \cap F(\Omega \cap$ $Q) \subset \mathcal{D}(T, \delta) \cap \mathcal{P}$ and $F(L \cap B \cap Q) \cap F(b \Omega \cap Q) \subset b \mathcal{D}(T, \delta) \cap \mathcal{P} \subset S \cap P(T, \delta)$ which implies that

$$
F(B \cap L \cap Q) \cap \mathcal{D}(T, \delta)=F(B \cap L \cap Q) \cap F(\Omega \cap Q)
$$

and

(3.16) $F(B \cap L \cap Q) \cap b \mathcal{D}(T, \delta)=F(B \cap L \cap Q) \cap F(b \Omega \cap Q) \subset S \cap P(T, \delta)$.

Clearly, $F(B \cap L \cap Q)$ intersects $S$ transversely.

Step 3. Choose $p \in F(L \cap B \cap Q) \cap F(b \Omega \cap Q)$. By (3.14) and by (F) the complex line $T$ passing through $p$ and $\tilde{w}_{0}$ belongs to $\mathcal{T}$ and is contained in some $H \in \mathcal{H}$ so by (E), $F(L \cap B \cap Q)$ intersects each complex hyperplane $H^{\prime}$ parallel to $H$ with $\operatorname{dist}\left(H, H^{\prime}\right)<2 \delta$ at precisely one point and transversely. Lemma 3.8 now implies that there is a biholomorphic map $\Phi$ from $H+2 \delta \mathbb{B}$ onto itself that fixes $H$ and maps $F(B \cap L \cap Q) \cap(H+2 \delta \mathbb{B})$ onto $p+2 \delta \Delta q$ where $q$ is a unit vector orthogonal to $H$. Note that $P(T, \delta) \subset \subset H+2 \delta \mathbb{B}$.

Let $U=\Phi(\mathcal{D}(T, \delta))$ and $\Sigma=\Phi(P(T, \delta) \cap S)=\Phi(b \mathcal{D}(T, \delta) \cap P(T, \delta))$. Now (3.15) and (3.16) imply that $\Phi(F(B \cap L \cap Q)) \cap(H+2 \delta \mathbb{B})=p+2 \delta \Delta q$ meets $\bar{U}$ in a domain bounded by a simple closed curve contained in $\Sigma \subset b U$, and the intersection is transverse. Moreover, since $\Phi$ fixes $H \supset T$ we have $p \in \Sigma \cap T$ and $T$ meets $\bar{U}$ in a domain bounded by a simple closed curve $\Sigma \cap T=S \cap T$ obtained as a transverse intersection of $T$ with $\Sigma \subset b U$.

With no loss of generality, assume that $p=0$. Let $M$ be the twodimensional complex subspace spanned by $T$ and $\mathbb{C} q$. Recall that $\mathbb{C} q$ meets $\bar{U}$ in a domain bounded by a simple closed curve which is a transverse intersection of $\mathbb{C} q$ with $\Sigma \subset b U$ and that $T$ intersects $\bar{U}$ in a domain bounded by a simple closed curve which is the transverse intersection of $T$ with $\Sigma \subset b U$. Since both $T \cap b U$ and $\mathbb{C} q \cap b U$ are contained in $\Sigma$, an open subset of $b U$ which is smooth, it follows that near $(T \cup b U) \cup(\mathbb{C} q \cap b U), b U$ is smooth and transverse to $M$. Thus $\tilde{U}=U \cap M$ is a bounded open set in $M$ which has smooth boundary near $(T \cap b U) \cap(\mathbb{C} q \cap b U)$. The component $\tilde{U}_{0}$ of $U$ containing $(T \cap U) \cup(\mathbb{C} q \cap U)$ is a bounded domain in $M$ which has smooth boundary near $\left(T \cap b \tilde{U}_{0}\right) \cup\left(\mathbb{C} q \cap b \tilde{U}_{0}\right)$ and which $T$ and $\mathbb{C} q$ meet in domains bounded by simple closed curves, which are transverse intersections of $T$ and $\mathbb{C} q$ with $b \tilde{U}_{0}$. Now apply Lemma 3.5 to get a sequence 
$\tilde{A}_{n} \subset \tilde{U}_{0}$ of transversely embedded analytic discs whose boundaries $b \tilde{A}_{n}$, in the sense of that lemma, converge to $\left(T \cap b \tilde{U}_{0}\right) \cup\left(\mathbb{C} q \cap b \tilde{U}_{0}\right)$. It is now clear that by pulling back to $b \Omega \cap Q$ with $(\Phi \circ F)^{-1}$ that $A_{n}=(\Phi \circ F)^{-1}\left(\tilde{A}_{n}\right)$, $A=(\Phi \circ F)^{-1}\left(T \cap U_{0}\right)$, and $L \cap B \cap Q \cap b \Omega=(\Phi \circ F)^{-1}(\mathbb{C} q \cap b \tilde{U})$ satisfy (3.13) and $w_{0} \in A, w_{0} \in A_{n}(n \in \mathbb{N})$.

It remains to show that everything can be done in such a way that $A_{n}(n \in$ $\mathbb{N}$ ) and $A$ belong to $\mathcal{D}$. By transversality, for each $T \in \mathcal{T}$ the $\operatorname{disc} T \cap F(Q \cap \Omega)$ is arbitrarily small perturbation of the intersection of $F(Q \cap \Omega)$ with $z_{1}$-axis provided that $\mathcal{T}$ is small enough. Further, our construction implies that the maps $\Phi$ are uniformly close to the identity provided that $\mathcal{H}$ and $\delta$ are small enough. Since $\mathcal{P}$ can be chosen arbitrarily small the reasoning from Discussion 3 following Lemma 3.3 applies to show that the discs $\tilde{A}_{n}$ belong to an arbitrarily small neighbourhood of $T \cap F(Q \cap \Omega)$ provided that $\mathcal{P}$ is small enough. It follows that $A_{n}, n \in \mathbb{N}$, and $A$ can be chosen to belong to $\mathcal{D}$.

Theorem 1.1 is thus proved in the case of convexity. It remains to prove it in the strictly pseudoconvex case.

To this end, observe first that in the proof of Lemma 3.6 we never used the fact that the elements of $\mathcal{L}$ are complex lines. What we needed was the following:

(a) There is an open neighborhood $\mathcal{W}$ of $z_{0}$ such that all $L \in \mathcal{L}_{0}$ are onedimensional complex submanifolds of $\mathcal{W}$,

(b) the initial $\Lambda\left(z_{0}\right) \in \mathcal{L}_{0}$ is tangent to $\mathcal{W} \cap b \Omega$,

(c) if $L \in \mathcal{L}_{0}$ is sufficiently close to $\Lambda\left(z_{0}\right)$ in the $\mathcal{C}^{1}$-sense and if $L$ meets $\mathcal{W} \cap \Omega$, then $L$ meets $b \Omega$ transversely in a simple closed curve bounding the domain $\Omega \cap L$, and

(d) given a neighborhood $\mathcal{V}$ of $z_{0}$, we have $L \cap \bar{\Omega} \subset \mathcal{V}$ provided that $L \in \mathcal{L}_{0}$ is sufficiently close to $\Lambda\left(z_{0}\right)$ in the $\mathcal{C}^{1}$-sense.

Now the following lemma is proved in exactly the same way as Lemma 3.6 .

Lemma 3.9. Let $\Omega$ be a bounded domain in $\mathbb{C}^{N}$ with $b \Omega$ of class $\mathcal{C}^{2}$. Suppose that $D_{0} \subset \Omega$ is a transversely embedded analytic disc. Let $w_{0} \in D_{0}$ and $z_{0} \in b D_{0}$. Let $\mathcal{W}, \Lambda\left(z_{0}\right)$ and $\mathcal{L}_{0}$ be as above. Let $\mathcal{D}$ be a neighbourhood of $D_{0}$ in the space of transversely embedded analytic discs. There is an $\mathcal{L} \subset \mathcal{L}_{0}$, a $\mathcal{C}^{1}$-neighbourhood of $\Lambda\left(z_{0}\right)$ in $\mathcal{L}_{0}$, such that for each $L \in \mathcal{L}$ that meets $\Omega \cap \mathcal{W}$

(a) $L \cap b \Omega$ is a compact convex curve, and

(b) there are $A \in \mathcal{D}$ and a sequence $\left\{A_{n}\right\}_{n=1,2, \ldots} \subset \mathcal{D}$ such that $w_{0} \in$ $A, w_{0} \in A_{n}(n \in \mathbb{N})$ and such that for each smooth 1 -form $\alpha$ on $\mathbb{C}^{2}$

$$
\lim _{n \rightarrow \infty} \int_{b A_{n}} \alpha=\int_{b A} \alpha+\int_{L \cap b \Omega} \alpha .
$$


To conclude the proof, we suppose that $b \Omega$ is strictly pseudoconvex at $z_{0}$. There exist a neighborhood $\mathcal{W}$ of $z_{0}$ and a biholomorphic map $\Psi: \mathcal{W} \rightarrow$ $\tilde{\mathcal{W}}, \mathcal{W}$ a neighborhood of $0=\Psi\left(z_{0}\right)$ such that $\Psi$ is a polynomial map of degree two and such that $\Psi(\Omega \cap \mathcal{W})$ is strictly convex. Let $\tilde{L}_{0}$ be the complex tangent line to $b \Psi(\Omega \cap \mathcal{W})$ at 0 . Let $\tilde{\mathcal{L}}_{0}$ be a neighborhood of $\tilde{L}_{0}$ in the space of complex lines, and for each $\tilde{L} \in \tilde{\mathcal{L}}_{0}$ let $L=\Phi^{-1}(\tilde{L} \cap \tilde{\mathcal{W}})$. If $\mathcal{W}$ and $\tilde{\mathcal{L}}_{0}$ are sufficiently small then $\mathcal{L}_{0}=\left\{L: \tilde{L} \in \tilde{\mathcal{L}}_{0}\right\}$ has the properties (a)-(d). Recall that by our assumptions, $\int_{b D} f \omega=0$ for every form $\omega \in \mathbb{C}^{1 ; 1}[d z]$ and for each transversely embedded analytic disc $D \in \mathcal{D}$. By Lemma 3.9 it follows that there is a neighborhood $\tilde{\mathcal{L}} \subset \tilde{\mathcal{L}}_{0}$ of $\tilde{L}_{0}$ in the space of complex lines and a neighborhood $Q$ of $z_{0}$ such that for each $L \in \mathcal{L}=\{L: \tilde{L} \in \tilde{\mathcal{L}}\}$ which meets $\Omega \cap \mathcal{W}$,

$$
\int_{L \cap b \Omega} f \omega=0
$$

for each $\omega \in \mathbb{C}^{1 ; 1}[d z]$. Since $\Psi$ is a polynomial map of degree two, it follows that for all $\alpha \in \mathbb{C}^{1 ; 0}[d z]$ the form $\Psi^{*} \alpha$ lies in $\mathbb{C}^{1 ; 1}[d z]$.

If $\tilde{L} \in \tilde{\mathcal{L}}$, then given a form $\alpha \in \mathbb{C}^{1,0}[d z]$ (3.18) implies that

$\int_{\tilde{L} \cap \Psi(b \Omega \cap \mathcal{W})}\left(f \circ \Psi^{-1}\right) \alpha=\int_{\Psi(L \cap b \Omega)}\left(f \circ \Psi^{-1}\right) \alpha=\int_{L \cap b \Omega}\left(f \circ \Psi^{-1} \circ \Psi\right) \Psi^{*} \alpha=0$.

By [GIS, Th. 3.2.1] it follows that $f \circ \Psi^{-1}$ is a CR-function in a neighborhood of 0 in $\Psi(b \Omega \cap \mathcal{W})$, which implies that $f$ is a CR-function in a neighborhood of $z_{0}$.

Theorem 1.1 is finally proved.

\section{Concluding Remarks.}

In this final section we note a consequence of the main theorem.

Let $\Omega$ be as in Theorem 1.1 and let $D \subset \Omega$ be a transversely embedded analytic disc. If a continuous function $f$ on $b D$ has a continuous extension to $\bar{D}$ which is holomorphic on $D$ then $\int_{b D} f \omega=0$ for all $(1,0)$-forms with linear coefficients. The holomorphic extendibility is invariant with respect to biholomorphic maps. Accordingly the following theorem holds in Stein manifolds, which we view as closed complex submanifolds of $\mathbb{C}^{M}$.

Theorem 4.1. Let $\Omega$ be a relatively compact domain in a closed complex submanifold $\mathcal{M}, \operatorname{dim} \mathcal{M} \geq 2$ of $\mathbb{C}^{M}$ that has $\mathcal{C}^{2}$ boundary. Let $D_{0} \subset \Omega$ be a transversely embedded analytic disc, and let $\Omega$ be strictly pseudoconvex at $z_{0} \in b D_{0}$. Let $\mathcal{D}$ be a neighbourhood of $D_{0}$ in the space of transversely embedded analytic discs $D \subset \Omega$. Suppose that $f$ is a continuous function on a neighbourhood of $b D_{0}$ in $b \Omega$ such that for each $D \in \mathcal{D}$ satisfying $w_{0} \in D$,

(4.1) $f \mid b D$ has a continous extension to $D$ which is holomorphic on $D$. 
Then $f$ is a CR function in a neighbourhood of $z_{0}$. If (4.1) holds for all $D \in \mathcal{D}$ then there are a neighbourhood $W$ of $D_{0}$ in $\mathcal{M}$ and a continuous function $\tilde{f}$ on $W \cap \bar{\Omega}$ such that $\tilde{f}=f$ on $W \cap b \Omega$.

Proof. Let $\operatorname{dim} \mathcal{M}=N$. The set $\bar{D} \subset \mathcal{M}$ is a compact, polynomially convex set in $C^{M}$ and thus has a Stein neighborhood basis in $\mathbb{C}^{M}$. The intersections of the elements of this basis with $\mathcal{M}$ form a Stein neighborhood basis of $\bar{D}$ in $\mathcal{M}$. Thus, by the result of Docquier and Grauert and the holomorphic triviality of holomorphic vector bundles on discs as used above and by the fact that the extendibility assumptions we make are invariant under biholomorhic maps, we can assume that $\Omega$ is a domain in $\mathbb{C}^{N}$ and that $D$ is the intersection of $\Omega$ with the $z_{1}$-axis. The preceding theorem now implies that $f$ is a CR-function in a neighborhood of $z_{0}$ in $b \Omega$. This proves the first part of the theorem. As $b \Omega$ is strictly pseudoconvex at $z_{0}$, there are a neighborhood $P$ of $z_{0}$ and a continuous function $\tilde{\tilde{f}}$ on $P \cap \bar{\Omega}$ that is holomorphic on $P \cap \Omega$ and that satisfies $\tilde{\tilde{f}}=f$ on $P \cap b \Omega$. Our assumptions imply that there is $\delta>0$ such that if $\mathcal{T}$ denotes the set of all complex lines parallel to the $z_{1}$-axis and at distance not exceeding $\delta$ from it, then each $T \in \mathcal{T}$ meets $P \cap b \Omega$ transversely (and thus also meets $P \cap \Omega$ ), $T$ meets $b \Omega$ in a simple closed curve bounding $T \cap \Omega$, and $f \mid(T \cap b \Omega)$ has a continuous extension $f_{T}$ to $T \cap \bar{\Omega}$ that is holomorphic in $T \cap \Omega$. Since $T \cap b \Omega \cap P$ contains an arc, $f_{T}$ coincides with $\tilde{\tilde{f}} \mid T$ near $b \Omega \cap P$. Thus, by Hartogs's lemma, the function $\tilde{f}$ defined by $\tilde{f} \mid(T \cap \bar{\Omega})=f_{T}$ has all the required properties on $\mathcal{W}=\cup\{T: T \in \mathcal{T}\}$.

This completes the proof.

\section{References}

[Al1] H. Alexander, Polynomial approximation and hulls in sets of finite linear measure in $C^{n}$, American J. Math., 93 (1971), 65-74.

[Al2] The polynomial hull of a rectifiable curve in $C^{n}$, American J. Math, 110 (1988), 629-640.

[G11] J. Globevnik, Testing analyticity on rotation invariant families of curves, Trans. Amer. Math. Soc., 306 (1988), 401-410.

[Gl2] _ _ A boundary Morera theorem in $C^{2}$, J. Geom. Anal., 3 (1993), 269-277.

[GlS] J. Globevnik and E.L. Stout, Boundary Morera theorems for holomorphic functions of several complex variables, Duke Math. J., 64 (1991), 571-615.

[GR] R.C. Gunning and H. Rossi, Analytic Functions of Several Complex Variables, Prentice-Hall, Englewood Cliffs, 1965.

[Po] Ch. Pommerenke, Boundary Behaviour of Conformal Maps, Springer-Verlag, BerlinHeidelberg-New York, 1992.

[Tu1] A.E. Tumanov, Extension of CR-functions into a wedge from a manifold of finite type (Russian), Mat. Sb. (N.S.), 136(178) (1988), 128-139. 
[Tu2] 334.

, Thin discs and a Morera theorem for CR functions, Math. Z., (1997), 327-

Received April 20, 1998. Research of the first author was supported in part by a grant from the Ministry of Science and Technology of the Republic of Slovenia. Research of the second author was supported in part by grant 9322326 from the National Science Foundation.

UNIVERSITY OF LJUBLJANA

LJUBLJANA

Slovenia

E-mail address: josip.globevnik@fmf.uni-lj.si

UNIVERSITY OF WASHINGTON

Seattle, WA 98195

E-mail address: stout@math.washington.edu 